\title{
A numerical method for analyzing fault slip tendency under fluid injection with XFEM
}

\author{
Daqing Liu ${ }^{1,2}$
}

Received: 8 September 2018/Accepted: 10 May 2019/Published online: 29 May 2019

(C) Springer-Verlag GmbH Germany, part of Springer Nature 2019

\begin{abstract}
We propose a numerical method for analyzing fault slip tendency under fluid injection using the extended finite element method (XFEM) both for fluid flow and poroelasticity. The fault is modeled as a zero-thickness interface, and we use a reduced model for the fluid flow in the fault to account for its hydraulic behavior. We use the rate- and state-dependent friction model as the fault friction model, and Biot's theory of poroelasticity to study the coupling between fluid flow and mechanical deformation in the surrounding porous media. Since a fully coupled method between fluid flow and poromechanics is computationally expensive, we have investigated the use of the so-called fixed-stress split in this context. In such a scheme, the fluid flow problem is solved firstly by freezing the total means stress field, and then the results are used to solve the mechanical problem. The fixed-stress split is unconditionally stable, consistent and more accurate for a given number of iterations compared with other type of splitting strategies. In order to verify our method, some test cases are presented. For the coupling between fluid flow and poromechanics, we consider the Terzaghi Problem and the Mandel Problem, comparing our results with those of previously published works. While, for the mechanic problem, we compare the results with those obtained using the software Pylith.
\end{abstract}

Keywords Coupling · Fault slip tendency · Fluid injection · Fluid flow · Fixed-stress split · Geomechanics · XFEM

\section{Introduction}

In several applications related, for instance, to the exploitation of oil and geothermal reservoirs and $\mathrm{CO}_{2}$ sequestration activities, the injection or extraction of fluids can alter the stress field in the reservoir, which could induce preexisting faults to reactivate (Fig. 1). Nowadays as a promising technology to reduce anthropogenic $\mathrm{CO}_{2}$ emissions into the atmosphere (e.g., [38, 42, 47, 50, $51,64])$, geologic storage of carbon dioxide $\mathrm{CO}_{2}$ draws more and more attention from researchers all around the world. In our research, we will focus on the reactivation of

Daqing Liu

liudaqing10@126.com

1 Institute of Mechanics, Chinese Academy of Sciences, Beijing, China

2 Modelling and Scientific Computing Lab (MOX), Department of Mathematics, Politecnico di Milano, Milan, Italy preexisting fault under fluid injection, because the reactivation of fault could produce a path for $\mathrm{CO}_{2}$ to leak, which plays a critical role to determine the success of geologic storage of carbon dioxide.

Fault reactivation induced by fluid injection is a complex problem including several parts such as the crustal deformation, fluid flow, coupling between fluid flow and mechanics, fault reactivation, and so on.

For the crustal deformation, boundary integral methods which have been used in dynamic simulations with complex geometry (e.g., [16, 39, 41, 65]) cannot simulate nonlinear bulk rheologies, and finite differences which have been used to simulate long, repeated rupture problems (e.g., [61-63]) lack the ability to deal with complex fault system geometry. While for the Finite Element Methods (FEM), although it could incorporate bulk rheology and nonplanar fault geometry (e.g., [1, 31]), it is very difficult to generate a mesh aligned with a nonplanar fault which is much more realistic in nature, especially when the fault geometry is uncertain such as in the fault reactivation and 


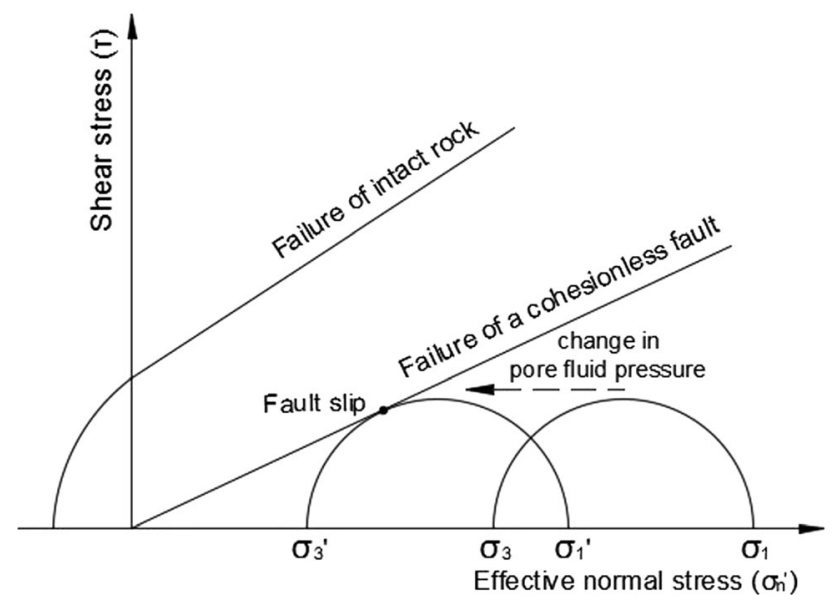

Fig. 1 Stress field affected by fluid injection

the mesh needs to change with the fault geometry is changed to study different possible scenario. So the eXtended Finite Element Method (XFEM) which was originally developed by Belytschko and colleagues (e.g., $[11,30,48])$ and modified by Fries [32], as a mesh-independent finite element method for embedded interfaces looks as a promising technique also for this clan of problems. XFEM extends the finite function space to include discontinuous functions across the fault, and it could deal with those discontinuities without remeshing (e.g., $[22,30,43,44,53])$.

For the interface conditions between the rock matrix and the fault, Lagrange multiplier methods and penalty methods are the most common methods. Set Lagrange multiplier methods is extremely mesh dependent and is not obviously extensible to three dimensions, and standard penalty methods typically result in ill-conditioned systems of equations [22]. So we have resorted to Nitsche's method, see Hansbo [36], which could be regarded as a hybrid of penalty methods and Lagrange multiplier methods, as our choice, because it provides a stabilized linear equations and is better conditioned than standard penalty methods. Moreover, we avoid saddlepoint problem complications inherent to Lagrange multiplier methods (e.g., $[6,7,10,22,35])$.

For the fluid flow, in D'Angelo et al. [25] the authors proposed an unfitted, mixed finite element method which views the fluid pressure and the fluid velocity as two variables of the system of equations for the Darcy's flows in fractured porous media. In their work, fractures were regarded as interfaces by using suitable reduced models (e.g., [3, 46]). A study of the well-posedness of XFEM method for flow in fractured porous media is found in Del Pra et al. [27].

For the coupling between fluid flow and geomechanics, various strategies have been investigated in the literature: fully coupled, iteratively coupled, explicitly coupled, and loosely coupled. In a fully coupled strategy, the governing equations are solved simultaneously at each time step, and a converged solution is obtained through iteration. Although the fully coupled strategy is unconditionally stable, it is computationally expensive [40]. In iteratively coupled scheme, one of the fluid flow problem and the mechanical problem will be solved firstly, and then the solution will be used to solve the other problem, the sequential procedure is iterated at each time step until the solution converges. By this scheme, the solution is the same as that obtained by the fully coupled method. Besides, this scheme could use different domains for the flow problem and the mechanical problem, which is useful because usually the domain of the mechanical problem is larger than that of reservoir simulation in order to deal with the reservoir boundary conditions (e.g., $[60,66])$. The explicitly coupled scheme is a special case of iteratively coupled scheme in which only one iteration is taken (e.g., $[8,9,52,69])$. In loosely coupled scheme, the coupling is resolved only after a certain number of flow time steps (e.g., [13, 26, 57]). Although this scheme can save computational cost, it is less accurate and requires reliable estimates of when to update the mechanical response [40]. Compared with the four strategies, the iteratively coupled scheme is a better choice for us.

For the iteratively coupled methods, there are four operator-split strategies: drained split, undrained split, fixed-strain split, and fixed-stress split. The drained split method solves the mechanical problem with frozen pressure field firstly, and then solves the fluid flow problem with frozen displacement field. Compared with the drained split method, the undrained split method allows pressure to change but keeps the fluid mass in each grid block remaining constant during the mechanical step. Fixedstrain split scheme solves the flow problem with frozen strain field, and then uses the solution to solve the mechanical problem. While, the fixed-stress split scheme solves the flow problem with frozen total means stress field firstly. From practical point of view, the framework for reservoir simulation could benefit from solving fluid flow problem firstly [40]. Besides, by analyzing these four operator-split strategies, Kim [40] found that the drained and the fixed-strain splits are only conditionally stable and are inconsistent if performed with a fixed number of iterations. While the undrained and the fixed-stress splits are unconditionally stable regardless of the coupling strength and are consistent even in the case of a single iteration per time step. For the undrained and the fixed-stress splits, the fixed-stress split is more accurate than the undrained split under the condition of a given number of iterations. Under his recommendation, we prefer to use the fixed-stress split as our scheme. 
For the fault reactivation, in the latest research [19], there are two methods used to represent the fault: one is employing solid elements in the fault aperture, and another one is using zero-thickness elements which act as an interface in the media. The previous one enables to describe accurately the hydraulic behavior of the fluid flow in the fault, but when we mainly study the slipping behavior of the fault in the process of reactivation, the latter one is much more effective. Fault reactivation may change the hydraulic aperture and the permeability of the fault, which will affect the hydraulic behavior of the fluid flow in the fault and in the surrounding media. In order to account for the hydraulic behavior in the fault and its effect on the surrounding media, in (e.g., [27, 46]), a reduced model of the fluid flow in the fault has been deduced, which we employ in our research. For the fault friction model, there are three types: static friction model, slipweakening model, and the rate- and state-dependent friction model, compared with these three models, the rateand state-dependent friction model is much more realistic, so we will use it as our fault friction model.

Compared with the previous research $[19,38,56]$ of the fault reactivation induced by fluid injection, we have chosen XFEM to deal with the discontinuities of the crustal deformation and the fluid flow across the fault. We regard the fault as a zero-thickness interface and use a reduced model to account for the hydraulic behavior in the fault. We use the Biot's theory of poroelasticity to study the coupling between fluid flow and mechanical deformation in porous media, and fixed-stress splitting scheme to deal with the coupling. We prefer to use the rate- and state-dependent friction model as our fault friction model. We start considering the two-dimensional map-view case, but in order to consider the dip of the fault, we will regard the system as a two-and-one-half-dimensional system based on Anderson's fault theory which assumes the vertical direction as principal stress component. Finally, we will do some test cases to verify our method.

\section{Governing equations}

In this work, we focus on the map-view two-dimensional case which means that we consider a two-dimensional domain that represents a top view of the three-dimensional domain.Firstly, we present the governing equations: the mechanic problem is governed by the equations for the balance of linear momentum, the fluid flow problem is governed by the equations for the balance of fluid mass and Darcy's law, and the coupling between mechanic problem and fluid flow problem is governed by the equations for the Biot's equations of poroelasticity.

\subsection{Balance of linear momentum}

The previous research has proved that the linear, constant coefficient elasticity is enough to describe many of the features of crustal deformation [22]. The momentum balance equation for the fully three-dimensional system governing displacement under isotropic linear elasticity is

$\nabla \cdot \sigma(\mathbf{u})=\rho \frac{\partial^{2} \mathbf{u}}{\partial t^{2}}$

where $\sigma$ is the total (Cauchy) stress tensor and $\mathbf{u}$ is the displacement.

In our research, we research on the fault reactivation under fluid injection, which process could be divided into two parts: the phenomena that occur before the fault reactivation, and the fault slipping behavior after the fault slips.

Firstly, we focus on the phenomena that occur before the fault slips to analyze the fault slip tendency under fluid injection. This process could be described using a quasistatic assumption which neglects the acceleration term in the momentum balance equation, because the time scale of this process is of the order of years.

In order to research on the map-view two-dimensional case, we simplify the equation to the so-called Mode III (anti-plane) deformation and Mode I/II (within-plane) deformation for a two-dimensional slice $\Omega$ of the threedimensional system [22], see Fig. 2.

For the map-view two-dimensional case, we use the Mode I/II (Mixed Mode) case in which deformation is only allowed to occur within the plane $\Omega$. Let us introduce the effective stress tensor $\sigma^{\prime}$ defined by

$\sigma^{\prime}=\sigma+b p \mathbf{I}$

where $\sigma$ is the total stress tensor, $b$ is the Biot coefficient, and $p$ is the fluid pressure.

Then the problem reads:

$$
\begin{aligned}
& \nabla \cdot \sigma(\mathbf{u})=\mathbf{0} \\
& \sigma^{\prime}(\mathbf{u})=\lambda \operatorname{tr}(\epsilon(\mathbf{u})) \mathbf{I}+2 \mu \epsilon(\mathbf{u}), \\
& \epsilon(\mathbf{u})=\frac{1}{2}\left(\nabla \mathbf{u}+(\nabla \mathbf{u})^{\mathbf{T}}\right) .
\end{aligned}
$$

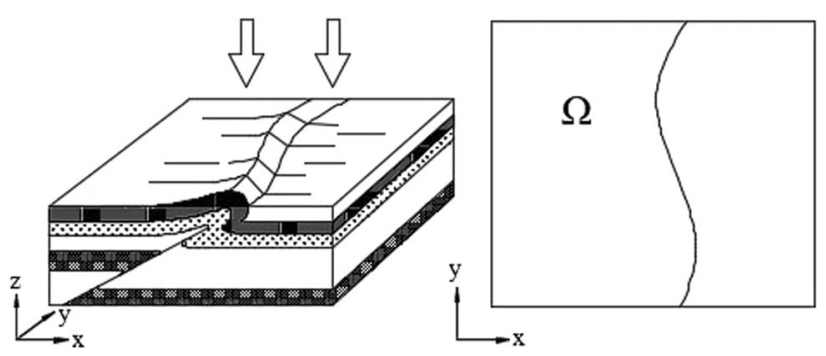

Fig. 2 Map-view case 
where $\mathbf{u}$ is the displacement, $\epsilon$ is the strain tensor, $\mathbf{I}$ is the identity tensor, $\lambda$ and $\mu$ are Lamé's parameters.

The governing equation for linear momentum balance of the porous medium becomes

$\nabla \cdot \sigma^{\prime}-b \nabla p=\mathbf{0}$ in $\Omega$

Boundary conditions will be detailed later on.

While for the slipping behavior after the fault slips, this process is a dynamic problem, and the momentum balance equation in the Mixed Mode case is:

$\nabla \cdot \sigma(\mathbf{u})=\rho \frac{\partial^{2} \mathbf{u}}{\partial t^{2}}$

and the governing equation for linear momentum balance of porous medium becomes:

$\nabla \cdot \sigma^{\prime}-b \nabla p=\rho \frac{\partial^{2} \mathbf{u}}{\partial t^{2}}$ in $\Omega$

\subsection{Balance of fluid mass}

For the isothermal single-phase fluid flow which is the focus of our research, the fluid mass conservation equation is

$\frac{\partial\left(\rho_{\mathrm{fl}} \phi\right)}{\partial t}+\nabla \cdot\left(\rho_{\mathrm{fl}} \mathbf{v}\right)=\rho_{\mathrm{fl}} f_{q}$,

where $\rho_{\mathrm{fl}}$ is the fluid density, $\phi$ is the porosity, $\mathbf{v}$ is the Darcy velocity, and $f_{q}$ is a volumetric source term.

We assume that fluid flow can be modeled by Darcy's law. Then, the relationship between the Darcy velocity $\mathbf{v}$ and the fluid pressure $p$ in the two-dimensional map-view case is

$\mathbf{v}=-\frac{\mathbf{k}}{\mu} \nabla p$

where $\mathbf{k}$ is the absolute permeability tensor, and $\mu$ is the fluid viscosity.

Here, we do not consider the variation of the fluid viscosity and define $\mathbf{K}=\mathbf{k} / \mu$. Then, the above equation becomes

$\mathbf{v}=-\mathbf{K} \nabla p$

\subsection{Biot's equations of poroelasticity}

According to Biot's theory of Poroelasticity [14, 24], we can express the fluid content variation in the porous medium as a function of pressure and volumetric strain as follows:

$\zeta=b \epsilon_{\mathrm{v}}+M^{-1} \delta p$ where $\zeta:=\delta m / \rho_{\mathrm{fl}, 0}=\delta \phi=\partial \phi / \partial t$ is the fluid content variation, $\epsilon_{\mathrm{v}}=\operatorname{tr}(\epsilon)$ is the volumetric strain, $M$ is the Biot modulus, and $\delta p$ denotes the pressure variation.

For the two-dimensional map-view case, the (rank-2) strain tensor could be expressed as:

$\epsilon=\frac{1}{2} \epsilon_{\mathrm{V}} \mathbf{I}+\mathbf{e}$

where $\mathbf{I}$ is the rank-2 identity tensor, and $\mathbf{e}$ is the deviatoric part of the strain tensor [24, 68].

\section{Boundary conditions}

In order to analyze the slipping behavior of the fault after the fault slips, we regard the fault as a zero-thickness interface. We assume that the domain $\Omega$ is a convex polygon. On the boundary, we will consider Dirichlet boundary conditions $\Gamma^{D}$ where displacement is imposed, Neumann boundary conditions $\Gamma^{N}$ where traction is imposed, and "Mixed" boundary conditions where displacement is only allowed to be imposed on the direction parallel to the boundaries. For the fluid flow problem, since we are considering the mixed formulation of the problem, normal velocity is imposed on the Dirichlet boundary $\Sigma^{D}$, while the boundary conditions on pressure are Neumann boundary conditions $\Sigma^{N}$.

For the boundary conditions of the mechanic problem, we use a far-field loading rate

$\mathbf{u}(t)=\mathbf{g}(t) \quad$ on $\Gamma^{D}$

to set Dirichlet boundary conditions on $\Gamma^{D}$, where $\mathbf{g}$ is a given function of time. For a constant velocity, assuming zero displacement at time 0 , we have $\mathbf{g}(t)=\mathbf{v}_{p} t$ in which $\mathbf{v}_{p}$ is the velocity on the boundaries. While for the Neumann boundaries, we set

$\sigma^{\prime} \cdot \mathbf{n}=\mathbf{F} \quad$ on $\Gamma^{N}$.

where $\mathbf{n}$ is the unit vector normal to the boundary, and $\mathbf{F}$ is a given traction.

For the fluid flow problem, we impose

$\left\{\begin{array}{cc}\mathbf{v} \cdot \mathbf{n}=\mathrm{v}_{0} & \text { on } \Sigma^{D}, \\ p=p_{0} & \text { on } \Sigma^{N},\end{array}\right.$

with $\Sigma^{D} \cup \Sigma^{N}=\partial \Omega, \Sigma^{D} \cap \Sigma^{N}=\emptyset$.

To satisfy the Dirichlet boundary conditions in the standard FEM, the solution $\mathbf{u}$ could be decomposed as $\mathbf{u}=\mathbf{u}_{0}+\mathbf{u}_{g}$, where $\left.\mathbf{u}_{0}\right|_{\Gamma^{D}}=0$ and $\left.\mathbf{u}_{g}\right|_{\Gamma^{D}}=\mathbf{v}_{p} t$. In order to eliminate the need for a particular solution $\mathbf{u}_{g}$, we prefer Nitsche's Method which enables essential boundary conditions to be implemented within the weak formulation by 
adding a consistent penalization term [17, 49]. Especially when we consider the fault, for the interface condition, the space is a function of time, by using Nitsche approach to impose interface condition on the fault, we do not need to discretize the space every time.

\section{Mathematical model of the fault}

In this work, we use a reduced model where the fault is represented by a line $\gamma$.

\subsection{Mechanic problem on the fault}

The fault $\gamma$ can be subdivided into two parts: the slipping region, that we denote as $\gamma^{N}$, where the two sides are allowed to have different displacements and we apply a friction force, and the stuck region $\left(\gamma^{D}\right)$ where a Dirichlettype boundary condition is used to impose continuity of displacements. Note that the two regions cover the whole $\gamma$ and may change in time due to modified mechanical conditions.

In the two-dimensional map-view case (Mixed Mode), the tangential component of slip is constrained by friction and failure, while normal component is set by a no-interpenetration constraint:

$\hat{\mathbf{t}} \cdot\left\{\sigma^{\prime}(\mathbf{u})\right\} \cdot \hat{\mathbf{n}}=f \quad$ on $\gamma^{N}$,

$\llbracket \mathbf{u} \rrbracket \cdot \hat{\mathbf{n}}=0 \quad$ on $\gamma$,

For the quasistatic problem:

$\llbracket \mathbf{u} \rrbracket \cdot \hat{\mathbf{t}}=g \quad$ on $\gamma^{D}$,

While for the dynamic problem:

$\llbracket \frac{\partial \mathbf{u}}{\partial t} \rrbracket \cdot \hat{\mathbf{t}}=0 \quad$ on $\gamma^{D}$

where $f$ is the friction traction detailed in the next section, $g$ is a given displacement jump on the tangential direction across the fault, $\hat{\mathbf{n}}$ is the unit vector normal to the fault, $\hat{\mathbf{t}}$ is the unit vector tangential to the fault, $\{\cdot\}$ and $\mathbb{I} \cdot \rrbracket$ indicate the average and the jump of a quantity which may be discontinuous across the fault correspondingly, and $\llbracket \mathbf{u} \rrbracket=\mathbf{u}^{+}-\mathbf{u}^{-},\{\mathbf{u}\}=\frac{1}{2}\left(\mathbf{u}^{+}+\mathbf{u}^{-}\right)$, the two sides of the fault being identified by + and - accordingly to Fig. 3 .

\subsubsection{Friction model}

Friction is taken equal to $f=\mu_{f} \sigma^{\prime}{ }_{n} \hat{f}$, where $\mu_{f}$ is the friction coefficient, $\sigma_{\mathrm{n}}^{\prime}=\hat{\mathbf{n}} \cdot \sigma^{\prime} \cdot \hat{\mathbf{n}}$, and $\hat{f}$ is the friction direction, which opposes the applied internal forces when

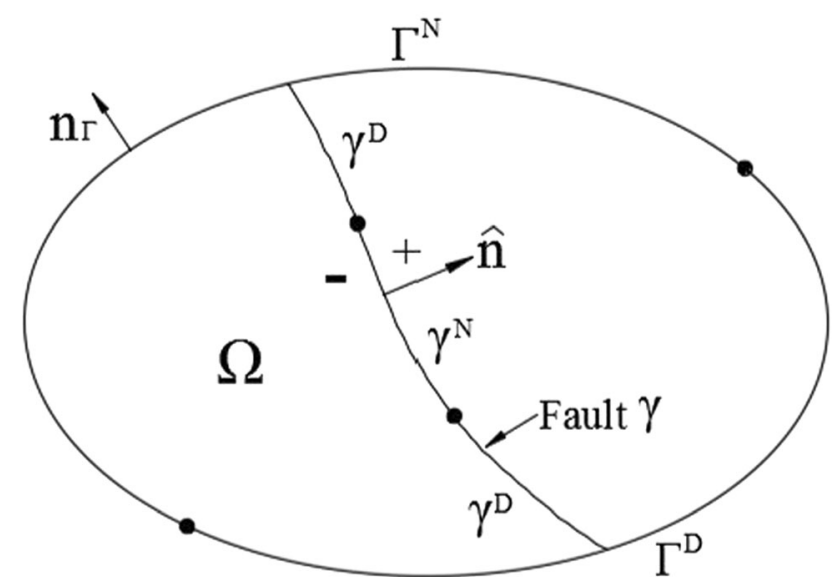

Fig. 3 The domain and the boundaries

the fault is stuck, while it opposes slip rate when the fault is slipping.

For the friction coefficient $\mu_{f}$, we choose the rate- and state-dependent friction model (Fig. 4) (e.g., $[28,29,38,45,55,58])$ :

$\mu_{f}=\mu_{0}+A \ln \left(\frac{V}{V_{0}}\right)+B \ln \left(\frac{V_{0} \theta}{d_{c}}\right)$,

$\frac{\mathrm{d} \theta}{\mathrm{d} t}=1-\frac{\theta V}{d_{c}}$,

where $V=\left|\frac{\mathrm{d}}{\mathrm{d} t} \mathbf{d}\right|$ is the slip rate magnitude, $\mathbf{d}=\llbracket \mathbf{u} \rrbracket \cdot \hat{\mathbf{t}}$ is the accumulated tangential slip, $\mu_{0}$ is the steady-state friction coefficient at the reference slip rate $V_{0}, A$ and $B$ are empirical dimensionless constants, and $\theta$ is the macroscopic variable characterizing state of the surface.

In this work, we use the Dieterich-Ruina rate and state friction model (a variational form of the rate- and statedependent friction model that prevent the risk of $V$ going to 0 ) [2] which is much more realistic as our fault friction model:

When $V \geq V_{\text {linear }}$,

$\mu_{f}=\mu_{0}+A \ln \left(\frac{V}{V_{0}}\right)+B \ln \left(\frac{V_{0} \theta}{d_{c}}\right)$

and when $V<V_{\text {linear }}$,

$\mu_{f}=\mu_{0}+A \ln \left(\frac{V_{\text {linear }}}{V_{0}}\right)+B \ln \left(\frac{V_{0} \theta}{d_{c}}\right)-A\left(1-\frac{V}{V_{\text {linear }}}\right)$.

When $\frac{V(t) \Delta t}{d_{c}}<\alpha_{v}$,

$\theta(t+\Delta t)=\theta(t) \exp \left(-\frac{V(t) \Delta t}{d_{c}}\right)+\Delta t-\frac{1}{2} \frac{V(t) \Delta t^{2}}{d_{c}}$

while when $V(t) \Delta t / d_{c} \geq \alpha_{v}$, 


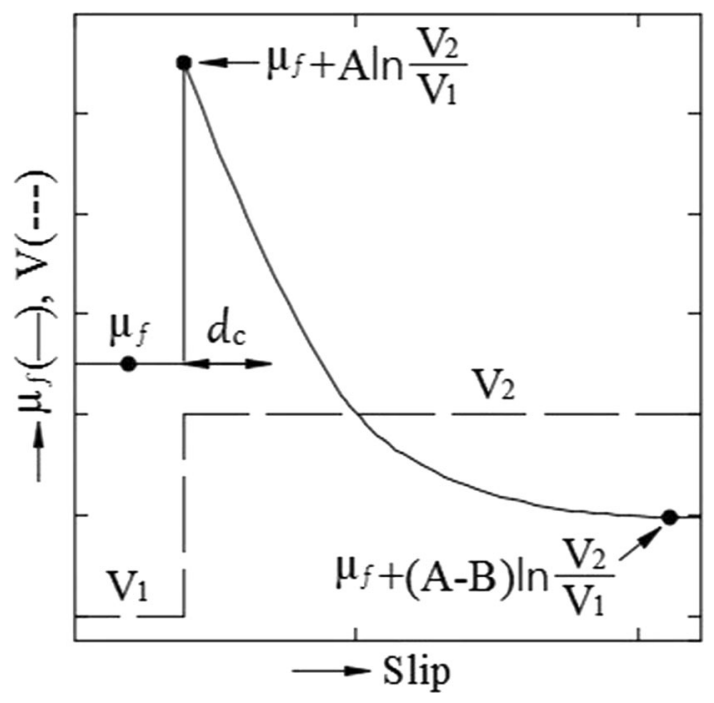

Fig. 4 Rate- and state-dependent friction model. The friction coefficient $\mu_{f}$ on the fault evolves with the slip rate or velocity $V$ and the state variable $\theta$. With the slip velocity increasing from $V_{1}$ to $V_{2}$ suddenly, the friction coefficient increases sharply due to a sudden increase in resistance from contact asperities firstly, and then declines slowly due to slip-weakening. The final steady-state value of the friction coefficient can be lower than the initial steady-state value if $A-B<0$, as shown in figure [38]

$$
\begin{aligned}
\theta(t+\Delta t)= & \theta(t) \exp \left(-\frac{V(t) \Delta t}{d_{c}}\right) \\
& +\frac{d_{c}}{V(t)}\left(1-\exp \left(-\frac{V(t) \Delta t}{d_{c}}\right)\right)
\end{aligned}
$$

where $V_{\text {linear }}$ is a cutoff for a linear slip rate dependence here taken equal to $10^{-12}$, and used to prevent failure due to null slip velocity $V=0$, and $\alpha_{v}$ is a small parameter here taken equal to $10^{-5}$.

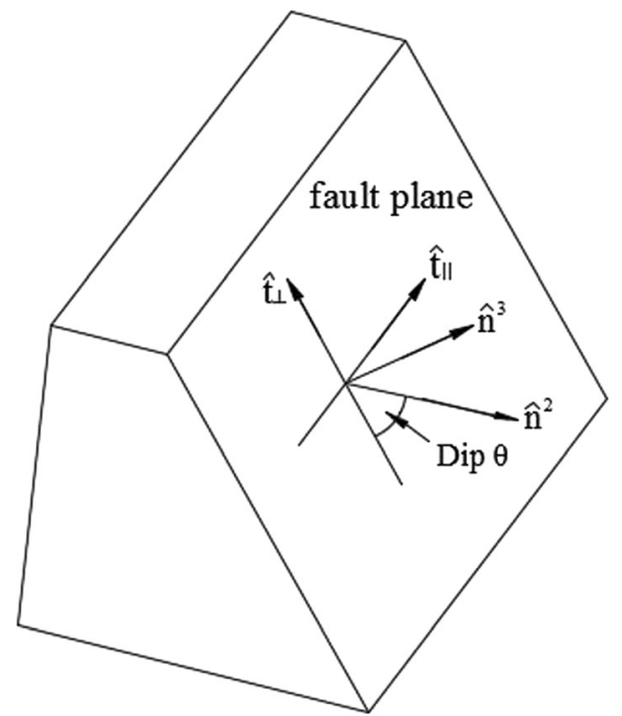

\subsubsection{Dipping fault}

In order to consider dipping faults (Fig. 5), we regard the system as a two-and-one-half-dimensional system based on Anderson's fault theory $[4,5]$ which assumes the vertical direction as principal stress component. We view the vertical stress as a constant, lithostatic value and assume that the vertical gradients can be neglected, and there is no variation in the fault along the dip [22].

The displacement $\mathbf{u}$ is a vector in the two-dimensional map-view layer, while the stress is a three-dimensional tensor in the two-and-one-half-dimensional system. By comparing the three-dimensional tractions on the fault plane to the fault strength, it could be determined whether the fault slips or not. If slip occurs, the three-dimensional traction is specified by friction, then the equivalent twodimensional stresses in the two-dimensional plane of the layer are determined [22].

Under the assumptions of this theory, the three-dimensional effective stress tensor on the fault is:

$\sigma^{\prime 3}=\left[\begin{array}{ccc}\sigma_{\tilde{x} \tilde{x}}^{\prime}+p_{l s} & \sigma_{\tilde{x} \tilde{y}}^{\prime} & 0 \\ \sigma_{\tilde{x} \tilde{y}}^{\prime} & \sigma_{\tilde{y} \tilde{y}}^{\prime}+p_{l s} & 0 \\ 0 & 0 & p_{l s}\end{array}\right]$

where $p_{l s}=\rho g d_{\Omega}$ is the lithostatic pressure on the layer. In this three-dimensional frame of reference, the effective normal stress $\sigma_{\mathrm{n}}^{\prime 3}=\hat{\mathbf{n}}^{3} \cdot \sigma^{\prime 3} \cdot \hat{\mathbf{n}}^{3}$, where the three-dimensional normal vector $\hat{\mathbf{n}}^{3}$ is given by $\hat{\mathbf{n}}^{3}=\left(\sin (\theta) \cdot \hat{\mathbf{n}}^{2}[0]\right.$, $\left.\sin (\theta) \cdot \hat{\mathbf{n}}^{2}[1], \cos (\theta)\right)^{T}$ for a fault with dip angle $\theta$. Besides, the effective shear stress has two components: $\hat{\mathbf{t}}_{\perp}=$ $\left(-\cos (\theta) \cdot \hat{\mathbf{n}}^{2}[0],-\cos (\theta) \cdot \hat{\mathbf{n}}^{2}[1], \sin (\theta)\right)^{T}$ and $\hat{\mathbf{t}}_{\|}=\left(-\hat{\mathbf{n}}^{2}\right.$ $\left.[1], \hat{\mathbf{n}}^{2}[0], 0\right)^{T}$.

In the fault plane, the three-dimensional effective traction is the vector $\tau^{\prime} \equiv\left(\tau_{\perp}^{\prime}, \tau_{\|}^{\prime}\right)$, where $\tau_{\perp}^{\prime}=\hat{\mathbf{t}}_{\perp} \cdot \sigma^{\prime 3} \cdot \hat{\mathbf{n}}^{3}$ and $\tau_{\|}^{\prime}=\hat{\mathbf{t}}_{\|} \cdot \sigma^{\prime 3} \cdot \hat{\mathbf{n}}^{3}$.

\subsubsection{Failure criteria}

We use a history-dependent criterion to check whether the fault will slip. A stuck point $\mathbf{x} \in \gamma^{D}$ begins to fail when the effective shear stress exceeds a critical friction value:

$\forall \mathbf{x} \in \gamma^{D} \quad$ satisfies $\quad \frac{\left|\tau^{\prime}(\mathbf{u}(\mathbf{x}))\right|}{\left|\sigma_{\mathrm{n}}^{\prime}(\mathbf{u}(\mathbf{x}))\right|} \geq \mu_{f}(\mathbf{x}) \quad \Rightarrow \quad \mathbf{x} \in \gamma^{N}$.

where $\mu_{f}$ is given by the friction model, and $\gamma^{N}$ is the portion of the fault where the failure criteria are met and the fault can slip. Here we define $\left|\tau^{\prime}(\mathbf{u}(\mathbf{x}))\right| /\left|\sigma^{\prime}{ }_{n}(\mathbf{u}(\mathbf{x}))\right|$ as the slip tendency of the fault.

Fig. 5 Dip fault 


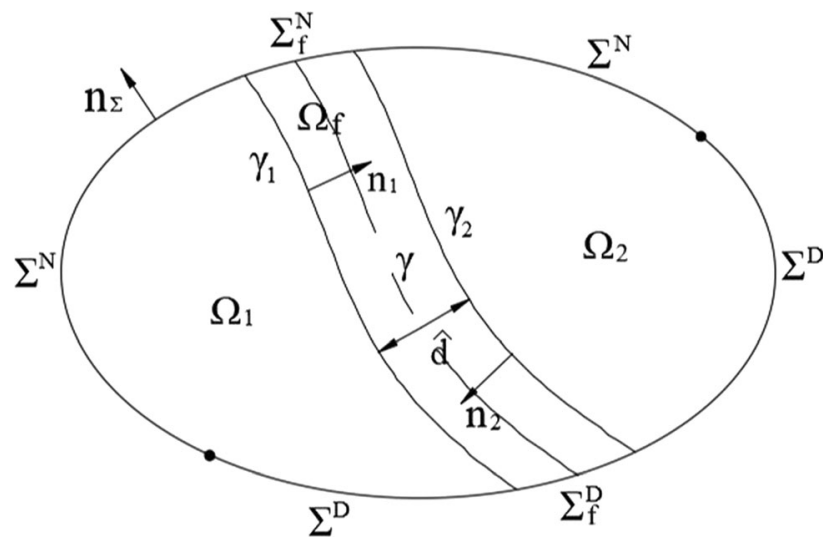

Fig. 6 The domain division [34]

\subsection{Reduced models for the fluid flow in the fault}

As shown in Fig. 6, the fault actually is a fault zone, in order to illustrate the model for fluid flow in the fault, we divide the whole domain into three parts: $\Omega_{i}, i=1,2, f . \Omega_{1}$ and $\Omega_{2}$ are the two parts of the original domain divided by the fault, while $\Omega_{f}$ is the fault area. In our research, we consider the fault as a zero-thickness interface. So we need to introduce the reduced models of the fluid flow in the fault $\gamma$ (e.g., [34, 46]):

1. Reduced conservation equation for the tangential component of the Darcy velocity in the fault becomes

$$
\frac{\partial \hat{d}}{\partial t}+\nabla_{\tau} \cdot \hat{\mathbf{v}}=\hat{f}_{q}+\llbracket \mathbf{v} \cdot \mathbf{n} \rrbracket \quad \text { on } \gamma,
$$

where $\hat{d}$ is the thickness of the fault, $\hat{f}_{q}$ is the total source in a section of the fault, $\hat{\mathbf{v}}$ is the Darcy velocity in the fault and $\nabla_{\tau}$. is the tangential divergence in the fault.

2. Reduced Darcy equation in the fault:

$$
\hat{\eta} \hat{\mathbf{v}}+\nabla_{\tau} \hat{p}=\mathbf{0} \text { on } \gamma,
$$

where $\hat{\eta}$ denotes the inverse of the equivalent permeability in the tangential direction to the fault $\gamma, \nabla_{\tau}$ is the tangential gradient, and $\hat{p}$ is the fluid pressure in the fault.

3. Reduced coupling conditions

$\eta_{\gamma}\{\mathbf{v} \cdot \mathbf{n}\}=\llbracket p \rrbracket \quad$ on $\gamma$

where $\eta_{\gamma}$ denotes the inverse of the equivalent permeability in the normal direction to the fault $\gamma$, and $\llbracket p \rrbracket$ denotes the jump of the fluid pressure in the porous medium across the fault.

4. Variation of pressure and velocity across the fault:

$$
\xi_{0} \eta_{\gamma} \llbracket \mathbf{v} \cdot \mathbf{n} \rrbracket=\{p\}-\hat{p} \quad \text { on } \gamma
$$

with $\xi_{0}$ is a closure parameter here taken equal to $1 / 8$. 5. Interface conditions of the fault:

$$
\left\{\begin{aligned}
\hat{p} & =\overline{\hat{p}} \quad \text { on } \partial_{N} \gamma \\
\hat{\mathbf{v}} \cdot \mathbf{n} & =\overline{\hat{\mathbf{v}}} \cdot \mathbf{n} \quad \text { on } \partial_{D} \gamma
\end{aligned}\right.
$$

\section{Coupled modeling of fluid flow and poromechanics}

We here recall the system of equations introduced in the previous parts in view of their coupling.

The domain $\Omega$ is split into two parts $\Omega_{1}$ and $\Omega_{2}$, separated by a fault described by the line $\gamma=\operatorname{int}\left(\bar{\Omega}_{1} \cap \bar{\Omega}_{2}\right)$.

We have adopted a quasistatic assumption for the rock mechanics. So, by setting $\Omega_{\gamma}=\Omega_{1} \cup \Omega_{2}$, we have that at any given time $t>0$,

$$
\left\{\begin{array}{l}
\nabla \cdot \sigma(\mathbf{u}, p)=\nabla \sigma^{\prime}(\mathbf{u})-b \nabla p=\rho \mathbf{f} \\
\frac{\partial\left(\rho_{\mathrm{f}} \phi\right)}{\partial t}+\nabla \rho_{\mathrm{f}} \mathbf{v}=\rho_{\mathrm{f}} f_{q} \quad \text { in } \Omega_{\gamma} \\
\mathbf{K}^{-1} \mathbf{v}+\nabla p=\rho_{\mathrm{f}} g \\
\sigma^{\prime}(\mathbf{u})=\lambda \operatorname{tr}(\epsilon(\mathbf{u})) \mathbf{I}+2 \mu \epsilon(\mathbf{u})
\end{array}\right.
$$

Porosity and permeability depend on the stress state. We assume that porosity variation is governed by

$$
\frac{\partial \phi}{\partial t}=b \epsilon(\dot{\mathbf{u}})+\frac{1}{M} \frac{\partial p}{\partial t}=b \nabla \cdot \dot{\mathbf{u}}+\frac{1}{M} \frac{\partial p}{\partial t} \quad \dot{\mathbf{u}}=\frac{\partial \mathbf{u}}{\partial t},
$$

where $M$ is the Biot modulus.

We choose to ignore the effects of water compressibility, so the second equation in Eq. (34) may be rewritten as $\frac{1}{M} \frac{\partial p}{\partial t}+\nabla(\mathbf{v}+b \dot{\mathbf{u}})=f_{q} \quad$ in $\Omega_{\gamma}$,

while for the permeability in the bulk, we use the model developed by Chin et al. [21]: $\mathbf{K}=\mathbf{K}_{0}\left(\phi / \phi_{0}\right)^{\alpha}$ where $\alpha \geq 1$ is a power-law exponent experimentally determined, and it depends on the type of rock considered, while $\mathbf{K}_{0}$ is the permeability at a reference porosity $\phi_{0}$. This model is justified by some experimental observations and allows us to describe the fact that a reduction of porosity induces a reduction of rock permeability. The system is supplemented by boundary conditions on $\partial \Omega_{\gamma}$, which correspond on fixing either the normal stress or the displacement on different portions of the boundary.

In the fault, the evolution of the tangential velocity flux $\hat{\mathbf{v}}$ obeys the model described in the previous parts, since we assume that variations of porosity in the fault for the 
phenomena under study are negligible. We recall it for completeness,

$$
\left\{\begin{array}{cc}
\nabla_{\tau} \cdot \hat{\mathbf{v}}=\hat{f}_{q}+\llbracket \mathbf{v} \cdot \mathbf{n} \rrbracket & \text { on } \gamma, \\
\hat{\eta} \hat{\mathbf{v}}+\nabla_{\tau} \hat{p}=\hat{\mathbf{f}}_{v}=\rho \mathbf{g} \cdot \hat{\mathbf{t}} & \text { on } \gamma,
\end{array}\right.
$$

with the boundary conditions on $\partial \gamma$ :

$$
\left\{\begin{array}{cl}
\hat{p}=\hat{p}_{N} & \text { on } \partial_{N} \gamma \\
\hat{\mathbf{v}} \cdot \mathbf{n}=\hat{\mathrm{v}}_{D} & \text { on } \partial_{D} \gamma
\end{array}\right.
$$

where $\hat{\mathrm{v}}_{D}$ and $\hat{p}_{N}$ are given data.

The effective permeability of the fault in the reduced model is given by $\hat{\eta}=\left(\mathrm{K}_{f, \tau} a\right)^{-1}$. However the material permeability $\mathrm{K}_{f, \tau}$ in the fault may vary with the state. In particular, even if we are assuming a compressive state and we do not have variation in the fault aperture due to normal displacements, we may have an effect related to shear dilation Ucar et al. [67]. To account for this effect we define an effective aperture $a_{\text {eff }}=a+b_{h s}$ where $b_{h s}$ is the aperture increase due to shear displacement, and it based determined according to Cappa et al. [20]. Then, we set $\mathrm{K}_{f, \tau}=\mathrm{K}_{f 0}\left(a_{\mathrm{eff}} / a_{0}\right)^{2}$, where $\mathrm{K}_{f 0}$ is a reference value at zero shear displacement.

\subsection{Coupling conditions}

We are left with the coupling conditions, which we write as follows

$$
\begin{cases}\xi_{0} \eta_{\gamma} \llbracket \mathbf{v} \cdot \mathbf{n} \rrbracket_{\gamma}=\{p\}-\hat{p}, & \\ \eta_{\gamma}\{\mathbf{v} \cdot \mathbf{n}\}_{\gamma}=\llbracket p \rrbracket_{\gamma}+a\left\{\mathbf{f}_{v} \cdot \mathbf{n}\right\}, & \\ \hat{\mathbf{t}} \cdot\left\{\sigma^{\prime}(\mathbf{u})\right\} \cdot \hat{\mathbf{n}}=f & \text { on } \gamma^{N}(t), \\ \llbracket \frac{\partial \mathbf{u}}{\partial t} \rrbracket \cdot \hat{\mathbf{t}}=0 & \text { on } \gamma^{D}(t), \\ \llbracket \mathbf{u} \cdot \hat{\mathbf{n}} \rrbracket=0 & \text { on } \gamma, \\ \llbracket \sigma \cdot \hat{\mathbf{n}} \rrbracket \cdot \hat{\mathbf{n}}=0 & \text { on } \gamma .\end{cases}
$$

where $\mathbf{f}_{v}$ is a generic forcing term, for instance due to gravity.

$\gamma^{N}(t)$ is defined as the partition of $\gamma$ where the ratio of tangential and normal stress exceeds a given value, more precisely, at any time $t>0$,

$\gamma^{N}(t)=\left\{\mathbf{x} \in \gamma: \frac{\left|\tau^{\prime}(\mathbf{x})\right|}{\left|\sigma_{n}^{\prime}(\mathbf{x})\right|}>\mu_{f}\right.$ or $\left.\left|\llbracket \frac{\partial \mathbf{u}}{\partial t} \rrbracket \cdot \mathbf{t}\right|(\mathbf{x})<\epsilon_{f}\right\}$,

where $\tau^{\prime}=\{\sigma \cdot \mathbf{n}\} \cdot \mathbf{t}$ and $\sigma_{n}^{\prime}=\{\sigma \cdot \mathbf{n}\} \cdot \mathbf{n}$ are the tangential and normal component of the average effective Cauchy stress on $\gamma$, respectively. We then set $\gamma^{D}(t)=\gamma \backslash \gamma^{N}(t)$. The first condition in Eq. (36) is the classic failure condition, the second condition allows a point in the slipping region $\gamma^{N}$ to remain in that region until its tangential velocity goes below a threshold value $\epsilon_{f}$. Here, $\mu_{f}$ is the friction coefficient, as described in previous parts.

\section{Time discretization}

Let us consider the time interval $(0, T)$ and define a partition $0=t_{0} \leq t_{1} \leq \cdots t_{N}=T$, with $N \in \mathbb{N}$. The time step $\Delta t_{k}=t_{k+1}-t_{k}$ is assumed to be constant and will be denoted simply as $\Delta t$. We introduce the semi-discretization in time of problem (Eq. (34) with the Implicit Euler method, reading

$$
\left\{\begin{array}{l}
\nabla \cdot \sigma^{\prime}\left(\mathbf{u}^{n+1}\right)-b \nabla p^{n+1}=0 \quad \text { in } \Omega_{\gamma} \\
\frac{1}{M} \frac{p^{n+1}-p^{n}}{\Delta t}+b \frac{\nabla \cdot \mathbf{u}^{n+1}-\nabla \cdot \mathbf{u}^{n}}{\Delta t}+\nabla \cdot \mathbf{v}^{\mathbf{n}+\mathbf{1}}=f_{q} \text { in } \Omega_{\gamma} \\
\nabla_{\tau} \cdot \mathbf{v}^{n+1}=\hat{f}_{q}+\llbracket \mathbf{v}^{n+1} \cdot \mathbf{n} \rrbracket \quad \text { on } \gamma
\end{array}\right.
$$

with the constitutive equations

$$
\left\{\begin{array}{c}
\sigma^{\prime}\left(\mathbf{u}^{n+1}\right)=\lambda \operatorname{tr} \epsilon\left(\mathbf{u}^{n+1}\right) \mathbf{I}+2 \mu \epsilon\left(\mathbf{u}^{n+1}\right) \\
\mathbf{K}\left(\mathbf{u}^{n+1}, p^{n+1}\right) \mathbf{v}^{n+1}+\nabla p^{n+1}=\mathbf{f}_{v} \\
K_{\tau}\left(\mathbf{u}^{n+1}\right) \hat{\mathbf{v}}^{n+1}+\nabla_{\tau} \hat{p}^{n+1}=\hat{\mathbf{f}}_{v}
\end{array}\right.
$$

and the coupling conditions [Eq. (35)] evaluated at time $t^{n+1}$. Note that the fluid and mechanics problems are coupled by some linear and nonlinear terms: the pressure gradient in the equilibrium equation, the divergence of the displacement in the mass conservation equation are linear coupling terms, while the dependence of permeability on porosity and effective aperture introduces nonlinearities in the system. Moreover, the interface conditions associated with the frictional contact are strongly nonlinear.

\subsection{Solution strategies for the coupled problem}

For the coupling between fluid flow and geomechanics, we prefer fixed-stress split which is unconditionally stable as our strategy. In fixed-stress split scheme, the fluid flow problem is solved firstly by freezing the total means stress field $\left(\dot{\sigma}_{\mathrm{v}}^{n+\frac{1}{2}}=\dot{\sigma}_{\mathrm{v}}^{n}\right)$. Then the original operator $\mathcal{A}$ could be decomposed as follows:

$$
\begin{gathered}
{\left[\begin{array}{c}
\mathbf{u}^{n} \\
p^{n}
\end{array}\right] \stackrel{\mathcal{A}_{s s}^{p}}{\longrightarrow}\left[\begin{array}{l}
\mathbf{u}^{n+\frac{1}{2}} \\
p^{n+1}
\end{array}\right] \stackrel{\mathcal{A}_{s s}^{\mathbf{u}}}{\longrightarrow}\left[\begin{array}{l}
\mathbf{u}^{n+1} \\
p^{n+1}
\end{array}\right], \quad \text { where }} \\
\left\{\begin{array}{c}
\mathcal{A}_{s s}^{p}: \dot{m}+\operatorname{Divv}=0, \delta \dot{\sigma}_{\mathrm{v}}=0, \\
\mathcal{A}_{s s}^{\mathbf{u}}: \operatorname{Div} \sigma=\mathbf{0}, \delta p=0 .
\end{array}\right.
\end{gathered}
$$

The ()$^{\dot{C}}$ denotes time derivative.

For the 2-dimensional map-view case, $\sigma_{\mathrm{v}}=\frac{1}{2} \operatorname{tr} \sigma$ is the volumetric part of the total stress tensor $\sigma$ :

$\sigma=\sigma_{\mathrm{v}} \mathbf{I}+\mathbf{s}$ 
where $\mathbf{S}$ is the deviatoric total stress tensor.

As already mentioned, in this scheme the flow problem is solved first assuming a constant ("frozen") mean stress field, and thus, a constant total volumetric stress. This allows, as we will show, to derive an expression for $\nabla$. $\mathbf{u}^{n+1}$ in the mass conservation equation.

Let the index $i$ denote the $i$ th iteration of the fixed-stress scheme. At time step $n+1$ we impose, for each iteration, that the total volumetric stress at iterations $i$ and $i-1$ are the same. Since $\sigma_{v}=K_{d r} \nabla \cdot \mathbf{u}-b p$, where $K_{d r}$ is the drained bulk modulus, equal to $\lambda+\mu$ in the two-dimensional case, we obtain

$K_{d r} \nabla \cdot \mathbf{u}^{n+1, i}-b p^{n+1, i}=K_{d r} \nabla \cdot \mathbf{u}^{n+1, i-1}-b p^{n+1, i-1}$.

Substituting Eq. (39) in the mass balance equation, we obtain

$$
\begin{aligned}
\frac{1}{\Delta t} & \left(\frac{1}{M}+\frac{b^{2}}{K_{d r}}\right) p^{n+1, i}+\nabla \cdot \mathbf{v}^{n+1, i} \\
= & f_{q}+\frac{1}{\Delta t M} p^{n}+\frac{b}{\Delta t} \nabla \cdot\left(\mathbf{u}^{n}-\nabla \cdot \mathbf{u}^{n+1, i-1}\right) \\
& +\frac{b^{2}}{K_{d r}} p^{n+1, i-1}
\end{aligned}
$$

This modified fluid problem does not depend on $\mathbf{u}^{n+1, i}$ and can be solved with the displacement fields at the previous time and at the previous iterations. The fixed-stress splitting algorithm can be summarized as follows:

Initialization At each time step $n+1$ set $\mathbf{u}^{n+1,0}=\mathbf{u}^{n}$ and $p^{n+1,0}=p^{n}$.

Fluid Subproblem Solve Eq. (40) coupled with the flow problem along the fault to obtain $p^{n+1, i}$ :

$$
\left\{\begin{array}{l}
\frac{1}{\Delta t}\left(\frac{1}{M}+\frac{b^{2}}{K_{d r}}\right) p^{n+1, i}+\nabla \cdot \mathbf{v}^{n+1, i}=f_{q}+\frac{1}{\Delta t M} p^{n} \\
\quad+\frac{b}{\Delta t} \nabla \cdot\left(\mathbf{u}^{n}-\nabla \cdot \mathbf{u}^{n+1, i-1}\right)+\frac{b^{2}}{K_{d r}} p^{n+1, i-1} \text { in } \Omega_{\gamma} \\
\mathbf{v}^{n+1, i}+\mathbf{K} \nabla p^{n+1, i}=\mathbf{f}_{v} \\
\nabla_{\tau} \cdot \hat{\mathbf{v}}^{n+1, i}=\hat{f}_{q}+\frac{1}{\Delta t} \llbracket \mathbf{v}^{n+1, i-1} \cdot \mathbf{n} \rrbracket \text { in } \gamma \\
\hat{\mathbf{v}}^{n+1, i}+K_{\tau} \nabla_{\tau} \hat{p}^{n+1, i}=\hat{f}_{v}
\end{array}\right.
$$

with coupling conditions on $\gamma$

$\left\{\begin{array}{c}\xi_{0} \eta_{\gamma} \llbracket \mathbf{v}^{n+1, i} \cdot \mathbf{n} \rrbracket=\left\{p^{n+1, i}\right\}-\hat{p}^{n+1, i}, \\ \eta_{\gamma}\left\{\mathbf{v}^{n+1, i} \cdot \mathbf{n}\right\}=\llbracket p^{n+1, i} \rrbracket+a\left\{\mathbf{f}_{v} \cdot \mathbf{n}\right\},\end{array}\right.$

as well as the given boundary conditions for the fluid problem.

Identification of $\gamma^{N}$ and $\gamma^{D}$ With the computed value of fluid pressure, we compute an intermediate total stress $\sigma=2 \mu \epsilon\left(\mathbf{u}^{n, i}\right)+\lambda \nabla \cdot \mathbf{u}^{n, i}-b \nabla p^{n+1, i}$ in order to estimate, with the help of (36), the extension of $\gamma^{D}$ and $\gamma^{N}$ at the current iteration, as well as the values of the friction coefficient.

Mechanic Subproblem Solve the equilibrium equation on $\Omega_{\gamma}$,

$$
2 \mu \epsilon\left(\mathbf{u}^{n+1, i}\right)+\lambda \nabla \cdot \mathbf{u}^{n+1, i}=b \nabla p^{n+1, i}
$$

together with the coupling interface conditions given by the last four equations in (35) and the given boundary conditions for the mechanical problem, to obtain $\mathbf{u}^{n+1, i}$. Convergence Test Test for convergence, then repeat steps 2 and 3 or proceed to the next time step. For the convergence we monitor $\left\|p^{n+1, i+1}-p^{n+1, i}\right\|_{L^{2}(\Omega)}$ and $\left\|\mathbf{u}^{n+1, i+1}-\mathbf{u}^{n+1, i}\right\|_{H^{1}\left(\Omega_{\gamma}\right)}$.

Remark 1 We point out that, as suggested in Both et al. [15], the coefficient $\beta=b^{2} / K_{d r}$ multiplying pressure in the fluid subproblem can be regarded as a stabilization parameter, thus, $\beta$ could take different values with an impact on the number of iterations needed to achieve convergence. In this work, we have adopted the classical, physically based value.

\section{Space discretization}

We can now introduce the space discretization strategy for the two problems: the fluid flow and the mechanics. In this work, we employ a finite element discretization, in particular, to achieve high flexibility in the presence of complex geometries, we resort to XFEM to represent interfaces across mesh elements.

\subsection{The grids}

In this work, we use the same mesh for the fluid flow problem and the geomechanical problem. This choice has the advantage of avoiding interpolation of material properties and variables, such as for instance the fluid pressure, between different grids. However, this is only possible because we are considering relatively simple synthetic cases: in real life applications the geomechanical model is usually larger than the fluid reservoir of interest and different grids are often used.

Both at the geomechanical and the reservoir scale the construction of a computational grid can be a challenging task in realistic configurations: indeed, grids should account for

- heterogeneous layers

- multiple and intersecting fractures and faults. 
Moreover, the fault surface is usually nonplanar and has a roughness that should be accounted for to compute reliable predictions of the slip tendency. In this work, we choose to employ nonconforming grids, i.e., we build the "bulk" mesh of the porous medium irrespective of the fault geometry and position. Thus, the domain $\Omega$ and the fault $\gamma$ are meshed independently.

We discretize the domain $\Omega$ into an approximation $\Omega_{h}$ which is a convex polygon and consider a family of triangulations $\mathcal{T}_{h}$. Let $h$ be the maximal diameter of the elements of $\mathcal{T}_{h}$. The approximation $\Omega_{h}$ is such that if $h$ goes to $0, \Omega_{h} \rightarrow \Omega$. In our examples, $\Omega$ is itself a polygon, so $\Omega_{h}=\Omega$. Analogously we define for $\gamma$ its piecewise linear approximation $\gamma_{h}$ (details will be given later on) and consider a family of triangulations $\mathcal{L}_{\hat{h}}$ (Fig. 7).

Remark 2 The mesh sizes $h$ and $\hat{h}$ can be chosen independently. However it has been observed, Frih et al. [33], that oscillations can arise in the fault if $\hat{h}<<h$. For this reason, we will use independent grid for the bulk and the fault, but with comparable mesh sizes.

Since $\mathcal{T}_{h}$ is not conformal with the fault $\gamma_{h}$, for the triangles $K \in \mathcal{T}_{h}$ cut by the fault $\gamma$, we define $K_{i}=K \cap \Omega_{i}$, where $\Omega_{i}, i=1,2$, are the two parts of the original domain divided by the fault $\gamma$, and use $\mathcal{G}_{h}=\left\{K \in \mathcal{T}_{h}: \gamma \cap K \neq \emptyset\right\}$ to denote the collection of the elements cut by the fault.

In the following we will assume, following D'Angelo et al. [25], that:

1. the triangulation is shape-regular, i.e., $c \rho_{K} \leq h_{K} \leq C \rho_{K}$ where $h_{K}$ is the diameter of element $K, \rho_{K}$ is the diameter of the largest circle contained in $K$ and $c, C$ are positive constants;

2. if $K \in \mathcal{G}_{h}$, then $\gamma$ intersects the boundary $\partial K$ twice exactly, and for each edge of $K$, it intersects at most one time;

3. $\gamma_{h}$ is "a good approximation" of $\gamma$, i.e., if $\gamma_{K}=\gamma \cap K$ and $\gamma_{K, h}=\gamma_{h} \cap K$ then $\gamma_{K}=\left\{(t, s): 0<s<\left|\gamma_{K, h}\right|, t=\right.$ $\delta(s)\}$ while $\gamma_{K, h}=\left\{(t, s): 0<s<\left|\gamma_{K, h}\right|, t=0\right\}$, where $\delta(s)$ is the distance between the fault $\gamma$ and the approximation $\gamma_{h}$.

The use of level sets to represent the fault

The geometry of the fault $\gamma$ is assumed to be given as a parametrized curve as $\gamma: \mathbf{x}=\mathbf{g}(s)$ with $s_{1} \leq s \leq s_{2}$. Let us define the signed distance of a point $\mathbf{x}$ from $\gamma$ as follows:

$\psi(\mathbf{x})=\min _{\mathbf{x}^{*} \in \gamma}\left|\mathbf{x}-\mathbf{x}^{*}\right| \operatorname{sign}\left(\mathbf{x}-\mathbf{x}^{*}\right) \cdot \mathbf{n}$

where $\mathbf{n}$ is the normal to the surface. Then $\gamma$ can be identified as the zero-level of $\psi$. We then replace $\psi$ with its finite element approximation (with linear finite elements) $\psi_{h}$ and obtain $\gamma_{h}$ as its piecewise linear zero-level set as shown in Fig. 8.

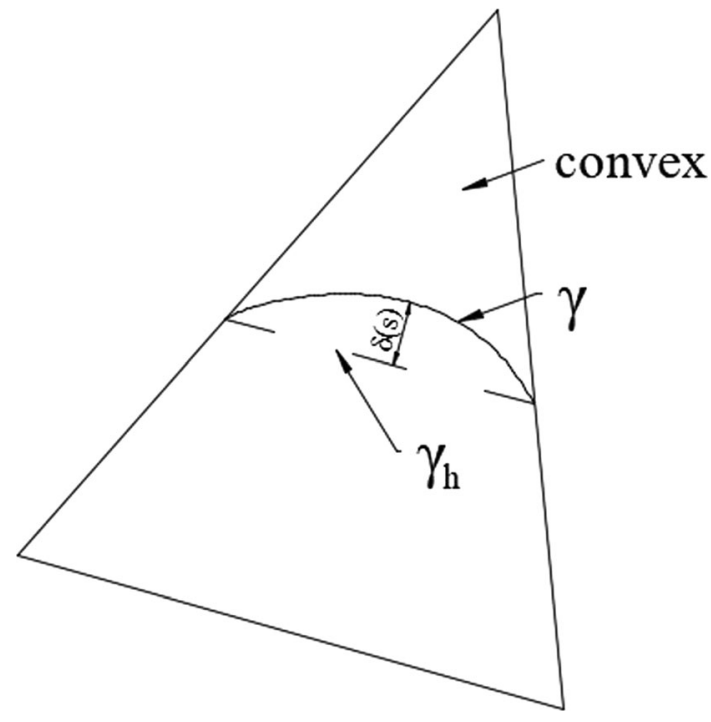

Fig. $7 \delta(s)$ is the distance between the curve fault $\gamma$ and the approximation $\gamma_{h}$ in the convex

Thus, the difference between $\gamma$ and $\gamma_{h}$ depends on the size of the bulk triangulation $\mathcal{T}_{h}$. This guarantees that if $\gamma$ has bounded curvature Assumption A-3 is always satisfied for a sufficiently refined grid.

The definition of the interface by means of a level set function is motivated by the capabilities of the finite element library Getfem++, used as the basis of our implementation, which provides the tool to define quadrature formulas on subdomains defined by the positive, negative or zero values of level sets.

\subsection{Space discretization with the XFEM}

As we mentioned in the previous sections the geometrical complexity of realistic cases motivates our choice of using nonmatching grids, i.e., to allow the fault to cut the elements of the bulk mesh. However, the material properties can change abruptly across the fault surface, and the solutions (pressure, fluid velocity and even displacement) can be discontinuous. For this reason, we employ the eXtended Finite Element Method (XFEM) to account for the discontinuities across the fault by means of suitable enrichments of some standard finite element spaces (Fig. 9) [11, 30, 48].

The XFEM is currently one of the most popular methods to discretize mechanical problems in linear elastic material in the presence of fractures, for industrial and geophysical applications. They have been applied to the problem of fault equilibrium and failure by E. Coon in Coon et al. [23] and Coon et al. [22]. In these works, however, the effect of fluid pressure and flow along and across the fault are not taken into account. The use of XFEM for the approximation of flow in porous media with fractures and faults is 

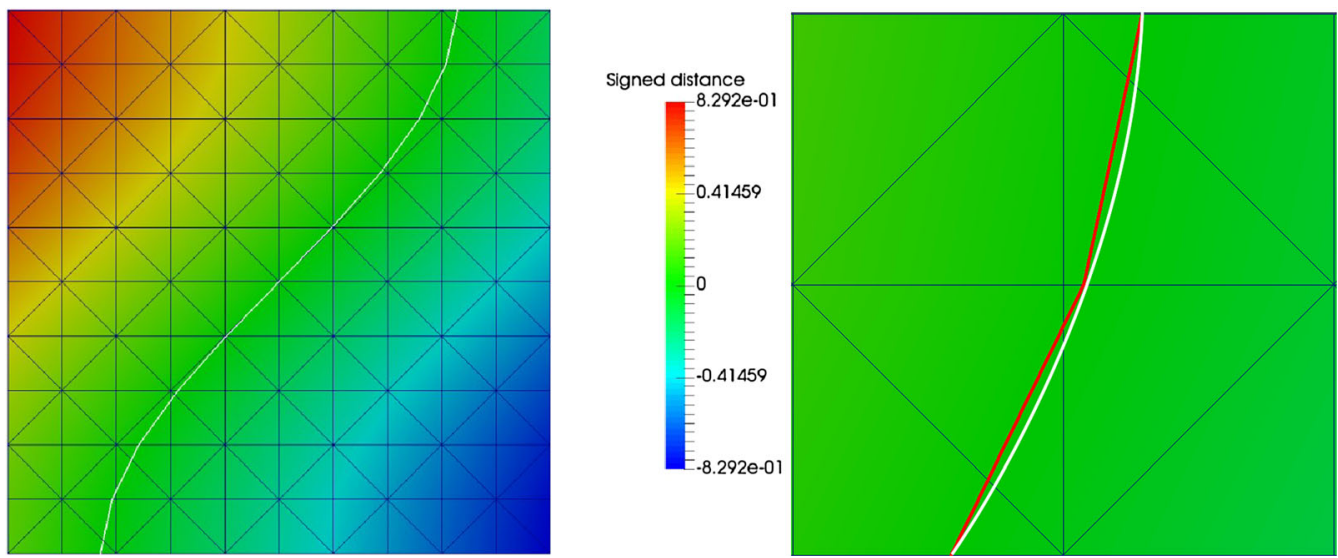

Fig. 8 The fault as the zero of a level set function equal to the signed distance from the interface (left) and a zoom of a detail (right). $\gamma$ is represented by the white line, and its approximation $\gamma_{h}$ by the red line

more recent but quite established, see for instance [25, 59] and Berrone et al. [12], where a single-phase Darcy flow is solved in undeformable fixed domains. In this work, we will discretize both the mechanical and fluid subproblems by means of the XFEM using the same nonmatching grid for the two problems to obtain a natural coupling and avoid interpolations.

\subsubsection{Flow problem}

For the numerical approximation of the flow problem in the bulk and along the fault we proceed as proposed in D'Angelo et al. [25] and Fumagalli [34]: starting from a stable mixed finite element pair for fluid velocity and pressure we enrich the finite element spaces to represent possible discontinuities across the fault. In particular, we start from the lowest order Raviart Thomas finite elements $\left(\mathbb{R} \mathbb{T}_{0}\right)$ for the Darcy velocity and piecewise constant $\left(\mathbb{P}_{0}\right)$ finite elements for pressure in the two-dimensional domain $\Omega_{h}$; analogously on the fault $\gamma_{h}$ we approximate the flux with $\mathbb{P}_{1}$ finite elements and the pressure with $\mathbb{P}_{0}$ elements. The choice of mixed finite elements is motivated by their property of local mass conservation and the robustness in the presence of permeability contrasts.

For each element $K \in \mathcal{T}_{h}$ we define the restriction of the standard $\mathbb{R} \mathbb{T}_{0}$ and $\mathbb{P}_{0}$ functions to the cut elements as follows:

$$
\begin{aligned}
\mathbb{R T}_{0}\left(K_{i}\right) & =\left\{\left.\mathbf{w}_{h}\right|_{K_{i}}: \mathbf{w}_{h} \in \mathbb{R T}_{0}(K)\right\} \\
\mathbb{P}_{0}\left(K_{i}\right) & =\left\{\left.q_{h}\right|_{K_{i}}: q \in \mathbb{P}_{0}(K)\right\} .
\end{aligned}
$$

Note that if $K \notin \mathcal{G}_{h}$ then $K_{i}$ either coincides with $K$ or it is empty. On each side of the fault, i.e., for $i=1,2$ we define the discrete spaces $\mathbf{W}_{i, h}, Q_{i, h}$ as

$$
\begin{aligned}
\mathbf{W}_{i, h} & =\left\{\mathbf{w}_{h} \in H_{\mathrm{div}}\left(\Omega_{i}\right):\left.\mathbf{w}_{h}\right|_{K_{i}} \in \mathbb{R} \mathbb{T}_{0}\left(K_{i}\right) \quad \forall K \in \mathcal{T}_{h}\right\}, \\
\mathbf{Q}_{i, h} & =\left\{q_{h} \in L^{2}\left(\Omega_{i}\right):\left.q_{h}\right|_{K_{i}} \in \mathbb{P}_{0}\left(K_{i}\right) \quad \forall K \in \mathcal{T}_{h}\right\} .
\end{aligned}
$$

We will consider discrete velocities $\mathbf{v}_{h}$ and pressures $q_{h}$ in the discrete spaces $\mathbf{W}_{h}=\mathbf{W}_{1, h} \times \mathbf{W}_{\mathbf{2}, \mathbf{h}}$ and $Q_{h}=Q_{1, h} \times$ $Q_{2, h}$ so that the variables are made by two independent components associated with the subdomains $\Omega_{i}$.

Remark 3 This formulation of the extended finite element spaces, where the test functions are restricted to the subelements and associated with different degrees of freedom on the two subdomains, is based on the approach proposed by Hansbo and Hansbo [36]. Equivalently, the standard finite element spaces can be enriched with the addition of discontinuous functions, see Belytschko et al. [11].

As concerns the discrete flux $\hat{\mathbf{w}}_{h}$ and fluid pressure $\hat{q}_{h}$ in the fault we define the following spaces:

$$
\begin{aligned}
& \hat{\mathcal{W}}_{i, h}=\left\{\hat{\mathbf{w}}_{h} \in L^{2}\left(\gamma_{h}\right):\left.\hat{\mathbf{w}}_{h}\right|_{l_{i}} \in \mathbb{P}_{1}\left(l_{i}\right) \quad \forall l \in \mathcal{L}_{h}\right\}, \\
& \hat{\mathcal{Q}}_{i, h}=\left\{\hat{q}_{h} \in L^{2}\left(\gamma_{h}\right):\left.\hat{q}_{h}\right|_{l_{i}} \in \mathbb{P}_{0}\left(l_{i}\right) \quad \forall l \in \mathcal{L}_{h}\right\} .
\end{aligned}
$$

The discrete weak formulation of the fluid problem (Eq. (41)) reads: given $\mathbf{u}_{h}^{n}, \mathbf{u}_{h}^{n+1, i-1}, p_{h}^{n}$, given the boundary data, find $\left(\mathbf{v}_{h}^{n+1, i}, \mathbf{v}_{h}^{n+1, i}, p_{h}^{n+1, i}, p_{h}^{n+1, i}\right) \in \mathbf{W}_{h} \times \hat{\mathbf{W}}_{h} \times Q_{h} \times$ $\hat{Q}_{h}$ satisfying, for all $\left(\mathbf{w}_{h}, \hat{\mathbf{w}}_{h}, q_{h}, \hat{q}_{h}\right) \in \mathbf{W}_{h} \times \hat{\mathbf{W}}_{h} \times Q_{h} \times$ $\hat{Q}_{h}$ :

$$
\begin{aligned}
& \mathcal{A}\left(\left(\mathbf{v}_{h}, \hat{\mathbf{v}}_{h}, p_{h}, \hat{p}_{h}\right),\left(\mathbf{w}_{h}, \hat{\mathbf{w}}_{h}, q_{h}, \hat{q}_{h}\right)\right)=\mathcal{F}\left(\mathbf{w}_{h}, \mathbf{q}_{h}\right) \\
& \quad \forall\left(\mathbf{w}_{h}, \mathbf{q}_{h}\right) \in \mathcal{D},
\end{aligned}
$$

where 


$$
\begin{aligned}
\mathcal{A}\left(\left(\mathbf{v}_{h}, \hat{\mathbf{v}}_{h}, p_{h}, \hat{p}_{h}\right),\left(\mathbf{w}_{h}, \hat{\mathbf{w}}_{h}, q_{h}, \hat{q}_{h}\right)\right) & \\
= & m\left(p_{h}^{n+1, i}, q_{h}\right)+c\left(\mathbf{v}_{h}^{n+1, i}, \mathbf{w}_{h}\right) \\
& +o\left(p_{h}^{n+1, i}, \mathbf{w}_{h}\right)-o\left(q_{h}, \mathbf{v}_{h}^{n+1, i}\right) \\
& +\hat{c}\left(\hat{\mathbf{v}}_{h}^{n+1, i}, \hat{\mathbf{w}}_{h}\right)+\hat{o}\left(\hat{p}_{h}^{n+1, i}, \hat{\mathbf{w}}_{h}\right)+ \\
& -\hat{o}\left(\hat{q}_{h}, \hat{\mathbf{v}}_{h}^{n+1, i}\right)+e\left(\hat{p}_{h}^{n+1, i}, \mathbf{w}_{h}\right)-e\left(\hat{q}_{h}, \mathbf{v}_{h}^{n+1, i}\right)
\end{aligned}
$$

and

$\mathcal{F}=R_{w}+R_{q}+\hat{R}_{w}+\hat{R}_{q}$.

with

$$
\begin{aligned}
& m\left(p_{h}, q_{h}\right)=\frac{1}{\Delta t} \int_{\Omega_{h}}\left(\frac{1}{M}+\frac{b^{2}}{K_{d r}}\right) p_{h} q_{h} \\
& c\left(\mathbf{v}_{h}, \mathbf{w}_{h}\right)=\int_{\Omega_{h}} \mathbf{K}^{-1} \mathbf{v}_{h} \cdot \mathbf{w}_{h}+\int_{\Sigma^{D}} \gamma_{0} h^{-1}\left(\mathbf{v}_{h} \cdot \mathbf{n}\right)\left(\mathbf{w}_{h} \cdot \mathbf{n}\right) \\
& +\int_{\gamma_{h}} \eta_{\gamma}\left\{\mathbf{v}_{h} \cdot \mathbf{n}\right\}_{\gamma}\left\{\mathbf{w}_{h} \cdot \mathbf{n}\right\}_{\gamma}+\xi_{0} \int_{\gamma_{h}} \eta_{\gamma} \llbracket \mathbf{v}_{h} \cdot \mathbf{n} \rrbracket_{\gamma} \llbracket\left[\mathbf{w}_{h} \cdot \mathbf{n} \rrbracket_{\gamma},\right. \\
& o\left(p_{h}, \mathbf{w}_{h}\right)=-\int_{\Omega_{h}} p_{h}\left(\nabla \cdot \mathbf{w}_{h}\right)+\int_{\Sigma^{D}} p_{h}\left(\mathbf{w}_{h} \cdot \mathbf{n}\right), \\
& \hat{c}\left(\hat{\mathbf{v}}_{h}, \hat{\mathbf{w}}_{h}\right)=\int_{\gamma_{h}} \hat{\eta} \hat{\mathbf{v}}_{h} \cdot \hat{\mathbf{w}}_{h}+\int_{\partial_{D} \gamma} \gamma_{0} h^{-1}\left(\hat{\mathbf{v}}_{h} \cdot \mathbf{n}\right)\left(\hat{\mathbf{w}}_{h} \cdot \mathbf{n}\right), \\
& \hat{o}\left(\hat{p}_{h}, \hat{\mathbf{w}}_{h}\right)=-\int_{\gamma_{h}} \hat{p}_{h}\left(\nabla_{\tau} \cdot \hat{\mathbf{w}}_{h}\right)+\int_{\partial_{D} \gamma} \hat{p}_{h}\left(\hat{\mathbf{w}}_{h} \cdot \mathbf{n}\right), \\
& e\left(\hat{p}_{h}, \mathbf{w}_{h}\right)=\int_{\gamma_{h}} \hat{p}_{h} \llbracket \mathbf{w}_{h} \cdot \mathbf{n} \rrbracket_{\gamma}
\end{aligned}
$$

and

$$
\begin{aligned}
R_{\mathrm{w}} & =\int_{\Omega_{h}} \mathbf{f}_{v} \cdot \mathbf{w}_{h}-\int_{\Sigma^{N}} \bar{p}\left(\mathbf{w}_{h} \cdot \mathbf{n}\right)+\int_{\Sigma^{D}} \gamma_{0} h^{-1} \bar{v}\left(\mathbf{w}_{h} \cdot \mathbf{n}\right) \\
& +\int_{\gamma_{h}} a\left\{\mathbf{f}_{v} \cdot \mathbf{n}\right\}_{\gamma}\left\{\mathbf{w}_{h} \cdot \mathbf{n}\right\}_{\gamma}-\int_{\gamma_{h}} \frac{a}{4} \llbracket \mathbf{f}_{v} \cdot \mathbf{n} \rrbracket_{\gamma} \llbracket\left[\mathbf{w}_{h} \cdot \mathbf{n} \rrbracket_{\gamma},\right. \\
R_{q} & =\frac{1}{\Delta t} \int_{\Omega_{h}}\left(\frac{1}{M} p_{h}^{n}+\frac{b^{2}}{K_{d r}} p_{h}^{n+1, i-1}\right) q_{h}+\int_{\Omega_{h}} b \nabla \\
& \left(\mathbf{u}_{h}^{n}-\mathbf{u}_{h}^{n+1, i-1}\right) q_{h}+\int_{\Omega_{h}} f_{q_{i}} q_{h}-\int_{\Sigma^{D}} \bar{u} q_{h}, \\
\hat{R}_{\mathbf{w}} & =\int_{\gamma_{h}} \hat{\mathbf{f}}_{v} \cdot \hat{\mathbf{w}}_{h}-\int_{\partial_{N} \gamma} \overline{\hat{p}}\left(\hat{\mathbf{w}}_{h} \cdot \mathbf{n}\right)+\int_{\partial_{D} \gamma} \gamma_{0} h^{-1} \overline{\hat{u}}\left(\hat{\mathbf{w}}_{h} \cdot \mathbf{n}\right), \\
\hat{R}_{q} & =\int_{\gamma_{h}} \hat{f}_{q} \hat{q}_{h}-\int_{\partial_{D} \gamma} \overline{\hat{u}} \hat{q}_{h},
\end{aligned}
$$

The problem can be written in matrix form as:

$$
\left[\begin{array}{cccc}
C & O & 0 & E \\
-O & 0 & 0 & 0 \\
0 & 0 & \hat{C} & \hat{O} \\
-E & 0 & -\hat{O} & 0
\end{array}\right]\left[\begin{array}{c}
\mathbf{V} \\
P \\
\hat{\mathbf{V}} \\
\hat{P}
\end{array}\right]=\left[\begin{array}{c}
R_{w} \\
R_{q} \\
\hat{R}_{w} \\
\hat{R}_{q}
\end{array}\right]
$$

where $E$ and $-E$ are the matrices corresponds to the coupling between the fluid flow problem and the geomechanics, $C, O$ and $-O$ are the matrices corresponds to the bulk, and $\hat{C}, \hat{O}$ and $-\hat{O}$ are the matrices corresponds to the fault.

\subsubsection{Mechanical problem}

The finite element space for the mechanical problem in the context of poroelasticity cannot be chosen independently from the flow problem for stability reasons. For the threefield formulation, i.e., flux, pressure and displacement, a common choice is to couple $\mathbb{R} \mathbb{T}_{0}-\mathbb{P}_{0}$ with linear finite element for displacement $\left(\mathbb{P}_{1}\right)$. This choice of spaces can successfully applied when the permeability tensor is uniformly positive definite; some issues have been observed on the case of vanishing permeability Rodrigo et al. [54]: volumetric locking may arise if the permeability is small with respect to the mesh size. Possible, more expensive alternatives are the quadratic finite elements or stabilized $\mathbb{P}_{1}$.

Analogously to what was done for the flow problem we define, for each element $K \in \mathcal{T}_{h}$ the restriction of the standard $\mathbb{P}_{1}$ as follows:

$\mathbb{P}_{1}\left(K_{i}\right)=\left\{\left.\phi_{h}\right|_{K_{i}}: \phi_{h} \in \mathbb{P}_{1}(K)\right\}$.

Then, bearing in mind that in our case the displacement $\mathbf{u}_{h}$ is a vector with two components, on each side of the fault we define the discrete spaces $\mathbf{Z}_{i, h}$ as

$$
\mathbf{Z}_{i, h}=\left\{\phi_{h} \in H^{1}\left(\Omega_{i}\right):\left.\phi_{h}\right|_{K_{i}} \in\left[\mathbb{P}_{1}\left(K_{i}\right]^{2}\right) \quad \forall K \in \mathcal{T}_{h}\right\} .
$$

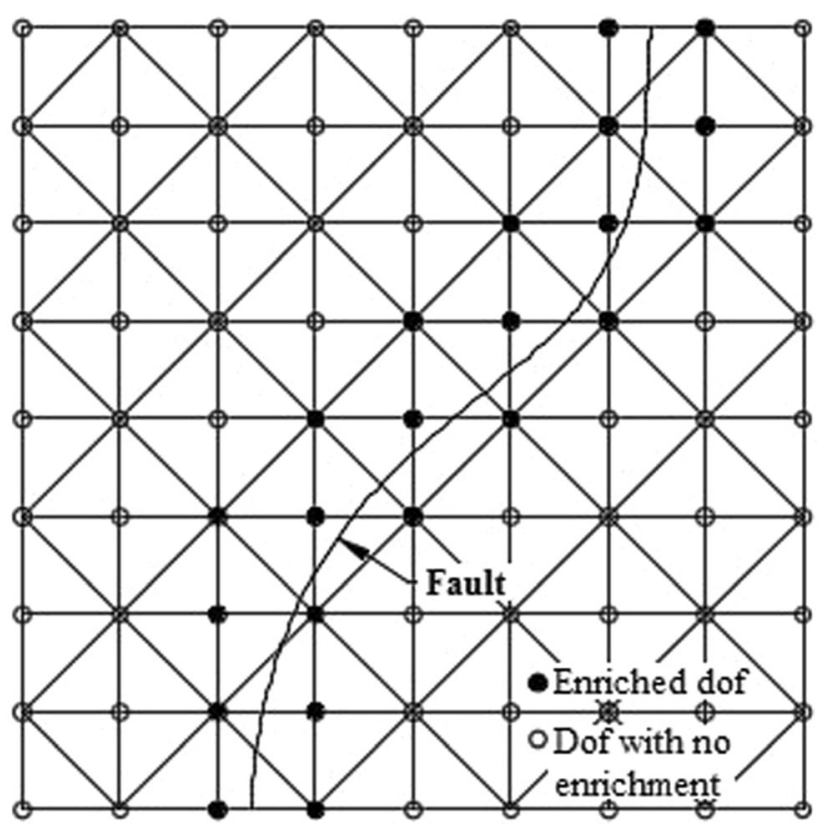

Fig. 9 Sketch of the mesh cut by the fault $\gamma$; the enriched elements are highlighted. The basis functions $\mathbb{P}_{1}\left(K_{i}\right)$ are represented 
We will consider discrete displacements $\phi_{h}$ in the discrete space $\mathbf{Z}_{h}=\mathbf{Z}_{1, h} \times \mathbf{Z}_{\mathbf{2}, \mathbf{h}}$ so that the variables are made by two independent components associated with the subdomains $\Omega_{i}$.

The discrete weak formulation of Eq. (42) reads: reads: given $p^{n+1, i}$ (computed in the fist "half" step of the fixedstress method), given the boundary data, find $\mathbf{u}_{h}^{n+1, i}, \in \mathbf{Z}_{h}$ satisfying, for all $\phi_{h} \in \mathbf{Z}_{h}$ :

$\mathcal{B}\left(\mathbf{u}_{h}, \phi_{h}\right)=\ell_{p}\left(\phi_{h}\right)$,

where

$$
\begin{aligned}
& \mathcal{B}\left(\mathbf{u}_{h}^{n+1, i}, \phi_{h}\right)=\int_{\Omega_{h}} \epsilon\left(\phi_{h}\right): \sigma^{\prime}\left(\mathbf{u}_{h}^{n+1, i}\right) \\
& \quad-\int_{\gamma_{h}}\left(\left(\llbracket \phi_{h} \rrbracket_{\gamma_{h}} \cdot \hat{\mathbf{n}}\right) \sigma_{\mathrm{n}}^{\prime}\left(\mathbf{u}_{h}^{n+1, i}\right)+\sigma_{\mathrm{n}}^{\prime}\left(\phi_{h}\right)\left(\llbracket \mathbf{u}_{h}^{n+1, i} \rrbracket_{\gamma_{h}} \cdot \hat{\mathbf{n}}\right)\right) \\
& \quad+\frac{\beta_{I}}{h} \int_{\gamma_{h}}\left(\llbracket \phi_{h} \rrbracket_{\gamma_{h}} \cdot \hat{\mathbf{n}}\right)\left(\llbracket \mathbf{u}_{h}^{n+1, i} \rrbracket_{\gamma_{h}} \cdot \hat{\mathbf{n}}\right) \\
& \quad+\frac{\beta_{D}}{h} \int_{\gamma^{D}}\left(\llbracket \phi_{h} \rrbracket_{\gamma^{D}} \cdot \hat{\mathbf{t}}\right)\left(\llbracket \mathbf{u}_{h}^{n+1, i} \rrbracket_{\gamma^{D}} \cdot \hat{\mathbf{t}}\right) \\
& \quad-\int_{\Gamma^{D}}\left(\phi_{h} \cdot \sigma^{\prime}\left(\mathbf{u}_{h}^{n+1, i}\right) \cdot \hat{\mathbf{n}}+\sigma^{\prime}\left(\phi_{h}\right) \cdot \hat{\mathbf{n}} \cdot \mathbf{u}_{h}^{n+1, i}\right) \\
& \quad+\frac{\beta_{\partial \Omega_{h}}}{h} \int_{\Gamma^{D}}\left(\phi_{h} \cdot \mathbf{u}_{h}^{n+1, i}+\left(\phi_{h} \cdot \hat{\mathbf{n}}\right)\left(\mathbf{u}_{h}^{n+1, i} \cdot \hat{\mathbf{n}}\right)\right) \\
& \ell_{p}\left(\phi_{h}\right)=\int_{\gamma^{N}}\left(\llbracket \phi_{h} \rrbracket_{\gamma^{N}} \cdot \hat{\mathbf{t}}\right) f+\frac{\beta_{D}}{h} \int_{\gamma^{D}}\left(\llbracket \phi_{h} \rrbracket_{\gamma^{D}} \cdot \hat{\mathbf{t}}\right) g^{n+1, i} \\
& \quad+\int_{\Gamma^{N}} \phi_{h} \cdot \mathbf{F}-\int_{\Gamma^{D}} \sigma^{\prime}(\phi) \cdot \hat{\mathbf{n}} \cdot \mathbf{g}\left(t_{n+1}\right) \\
& \quad+\frac{\beta_{\partial \Omega_{h}}}{h} \int_{\Gamma^{D}}\left(\phi_{h} \cdot \mathbf{g}\left(t_{n+1}\right)+\left(\phi_{h} \cdot \hat{\mathbf{n}}\right)\left(\mathbf{g}\left(t_{n+1}\right) \cdot \hat{\mathbf{n}}\right)\right) \\
& \quad+b \int_{\partial \Omega_{h}} \tilde{p} \phi_{h} \cdot \mathbf{n}-b \int_{\Omega_{h}} p^{n+1, i} \nabla \cdot \phi_{h} .
\end{aligned}
$$

Note that the pressure term on the right-hand side has been integrated by parts and here $\tilde{p}$ denotes a suitable extension to the boundary of the known pressure field $p^{n+1, i}$.

The imposed tangential displacement jump on the stuck portion of the fault is denoted as $g^{n+1, i-1}$ and is taken from the previous iteration of the fixed-stress method, i.e., we set $g^{n+1, i}=\llbracket \mathbf{u}^{n+1, i} \rrbracket \cdot \hat{\mathbf{t}}$.

Remark 4 The coupling conditions on the Dirichlet portion of the interface $\gamma_{D}$, i.e., the essential condition on the displacement jump across the fault is imposed with a consistent Nitsche penalization. As already mentioned in Chapter 3 this strategy has the advantage of enforcing the interface conditions on both $\gamma_{N}$ and $\gamma_{D}$ in the weak formulation and not in the discrete functional spaces. Since the partition of $\gamma$ into the Neumann (slipping) part and the Dirichlet (stuck) part can change in time the use of Nitsche

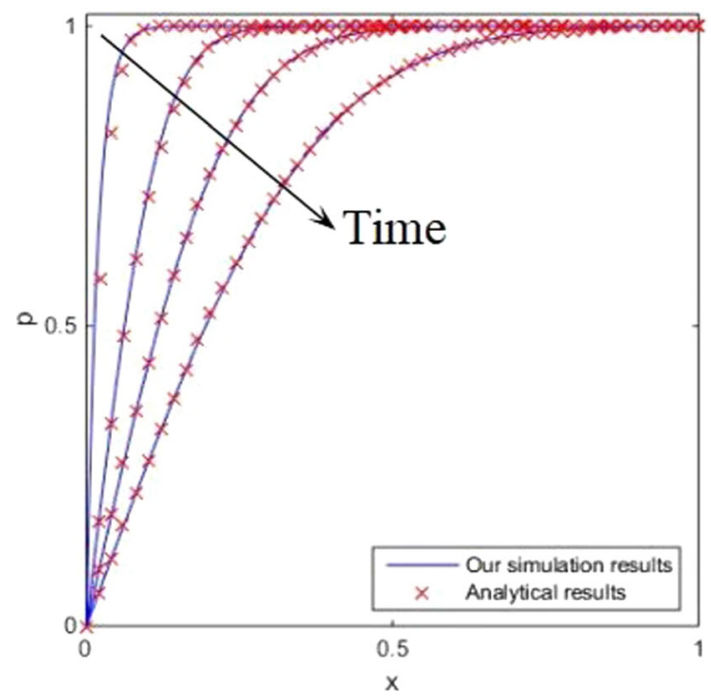

Fig. 10 Terzaghi Problem: pressure against distance at four different times

penalization avoids changing the discrete spaces at each time step.

Remark 5 The choice of $\gamma / h^{\alpha}$ with $\alpha=1$ as Nitsche penalization parameter leads to optimal error estimates in the case of our interest, Burman et al. [17, 18]. Other values of $\alpha$ can be chosen in the case of solutions with higher regularity and quadratic Lagrangian finite elements.

\section{Verification and validation}

In order to verify our proposed method, we did some test cases.

\subsection{Test 1: the Terzaghi problem}

We consider the Terzaghi's uniaxial compaction problem. Parameters are set as in Jha et al. [38]: size of the domain is $1 \mathrm{~m} \times 50 \mathrm{~m}$, the bottom boundary is fixed and impermeable, on the left and the right boundaries, the displacement is only allowed in the y-direction without fluid flow, while the top boundary is a Neumann boundary with a compression of $2.125 \mathrm{MPa}$ and the pressure is set to 0. The Young's modulus $E$ is $120 \mathrm{MPa}$, and the drained Poisson ratio is 0.3 , Biot coefficient is 1.0 , porosity is 0.2 , and hydraulic diffusivity is $1.9 \times 10^{-6} \mathrm{~m}^{2} / \mathrm{s}$. Results are shown in Fig. 10 .

\subsection{Test 2: the Mandel problem}

We consider the Mandel's problem. Parameters are set as in Jha et al. [38]: size of the domain is $50 \mathrm{~m} \times 10 \mathrm{~m}$ which is different from that in Jha et al. [38], because we consider the two-dimensional map-view case, so we do not consider 


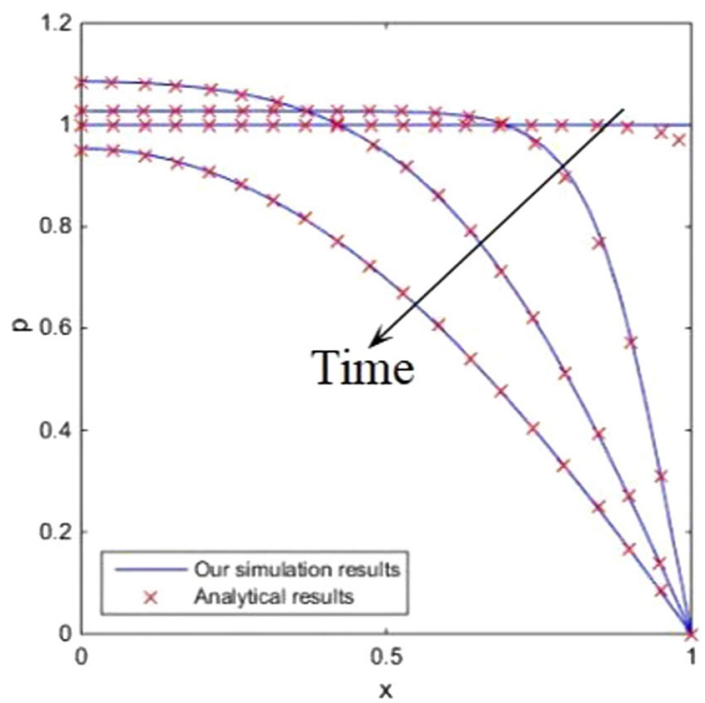

Fig. 11 Pressure evolution of the Mandel Problem

the thickness, on the right and the bottom boundaries displacement is only allowed on the direction parallel to the boundaries and the boundaries are impermeable, while the top boundary is a Neumann boundary with no stress and pressure equals to 0 , the left boundary is a Neumann boundary with a compression $1 \mathrm{MPa}$ with no fluid flow. The Young modulus $E$ is $18 \mathrm{GPa}$, drained Poisson ratio is 0.25 , undrained Poisson ratio is 0.49 , reference porosity is 0.05 , and hydraulic diffusivity is $2.2 \times 10^{-7} \mathrm{~m}^{2} / \mathrm{s}$. Results are shown in Fig. 11.

\subsection{Test 3: geomechanical problem with a fault}

In this section we compare our results with the results provided by Pylith [37] on a purely geomechanical problem without the fluid. We consider a two-dimensional mapview domain. In this case, each time step is 2.0 years and the total time is 300 years. The relevant parameters are in Table 1. The domain is shown in Fig. 12, the size is

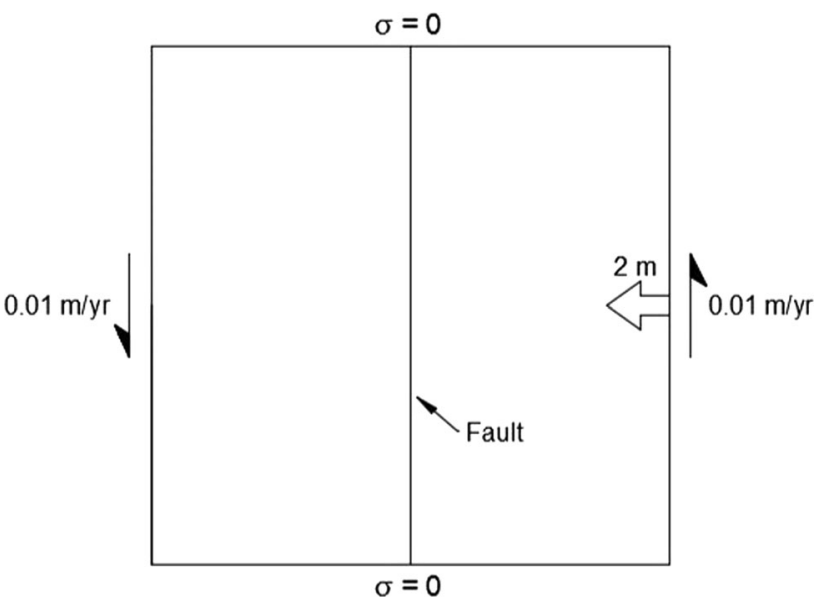

Fig. 12 Domain and boundary conditions for test case of geomechanical problem with a fault

$6000 \mathrm{~m} \times 6000 \mathrm{~m}$. The fault is represented by a straight line from point $(3000,0)$ to point $(3000,6000)$. On the right boundary, there is an initial displacement of $2 \mathrm{~m}$ normal to the boundary. The left and right boundaries are all Dirichlet boundaries with a constant velocity $0.01 \mathrm{~m} / \mathrm{yr}$ parallel to the boundaries. The other boundaries are all Neumann boundaries with null traction. As shown in Fig. 13, our results are in good agreement with those by Pylith: in particular, we have reported the slip magnitude and the shear traction at the center of the fault. In Fig. 14, the displacement on the $y$-direction after 145th step (290 years) done by Pylith and our code are shown, respectively, the results match very well. The difference of the slip magnitude and the shear traction after fault slips in the figure is because after we got the displacement result in the bulk mesh without the fault, in the postprocessing we got a new displacement result in a new mesh which includes the fault, then we got the slip magnitude based on the difference of the new displacement result on two new degree of freedom on the two sides of the fault, even if the two new degree of freedom are very close, there is still a

Table 1 Relevant parameters of mechanic problem

\begin{tabular}{lll}
\hline Variable & Value & Description \\
\hline$\rho$ & $2500 \mathrm{~kg} / \mathrm{m}^{3}$ & Density \\
$\lambda$ & $25 \mathrm{GPa}$ & Lamé's parameter \\
$\mu$ & $22.5 \mathrm{GPa}$ & Shear modulus \\
$\mu_{0}$ & 0.4 & Reference friction coefficient \\
$V_{0}$ & $1.0 \mathrm{e}-11 \mathrm{~m} / \mathrm{s}$ & Reference slip rate \\
$d_{c}$ & $0.05 \mathrm{~m}$ & Characteristic slip distance \\
$A$ & 0.002 & Empirical dimensionless constants \\
$B$ & 0.08 & Empirical dimensionless constants \\
$V_{\text {linear }}$ & $1.0 \mathrm{e}-9$ & A cutoff for a linear slip rate dependence
\end{tabular}



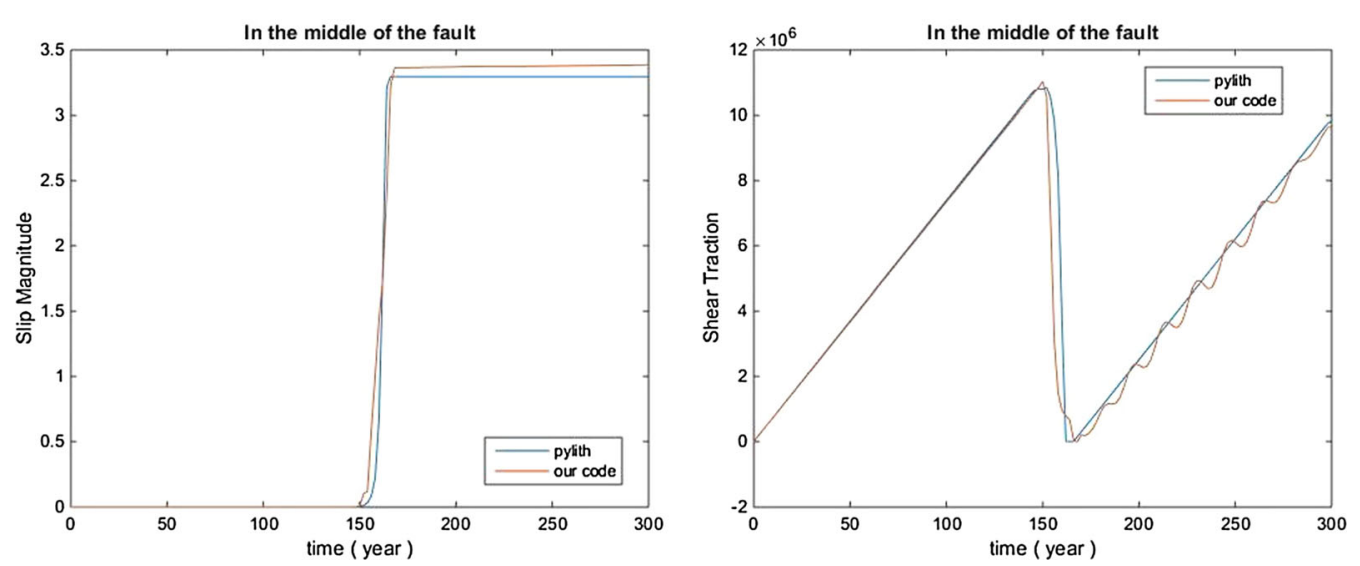

Fig. 13 Slip magnitude and shear traction in time in the middle of the fault
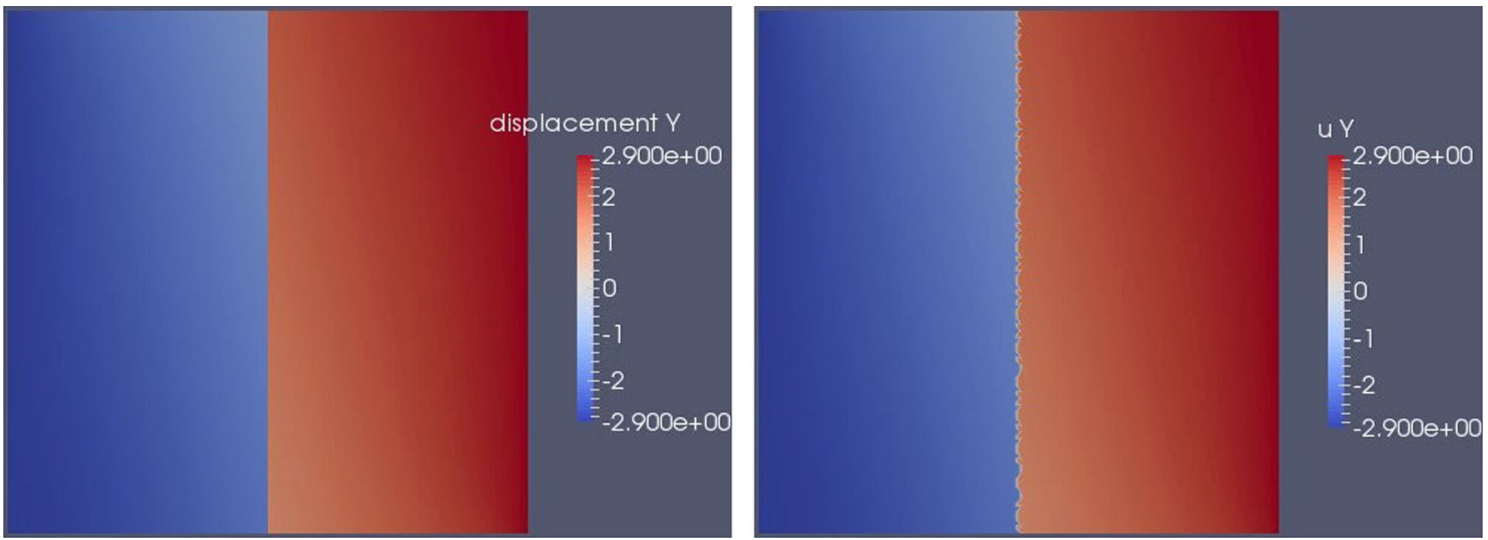

Fig. 14 Displacement on the $y$-direction after the 145th step (290 years) done by Pylith and our code

distance between them which will affect the result of slip magnitude and then affect the result of shear traction.

The fault begins to slip after the 74th step (148th year). The friction coefficient $\mu_{f}$ and the slip tendency $\left|\tau^{\prime}(\mathbf{u}(\mathbf{x}))\right| /\left|\sigma_{\mathrm{n}}^{\prime}(\mathbf{u}(\mathbf{x}))\right|$ on the fault after the 73rd step (146th year) and the 74th step (148th year) are shown in Fig. 15 correspondingly.

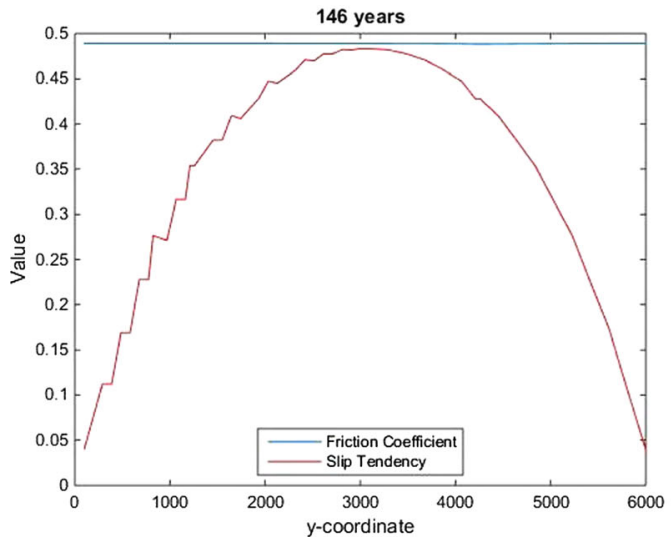

\section{Applications: slip tendency of the fault under fluid injection}

In this example, we analyze the slip tendency of the fault under fluid injection. As shown in Fig. 16, the size of the domain is $4000 \times 3000 \mathrm{~m}$, on the behind boundary and the right-hand side boundary, we only allow the displacement to occur in the direction parallel to the boundaries, while on

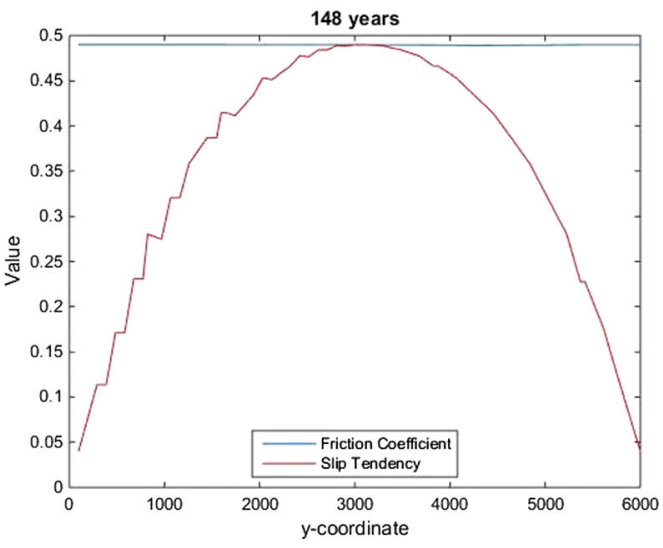

Fig. 15 Friction coefficient and slip tendency on the fault after the 146th year (73th step) and the 148th year (74th step) 


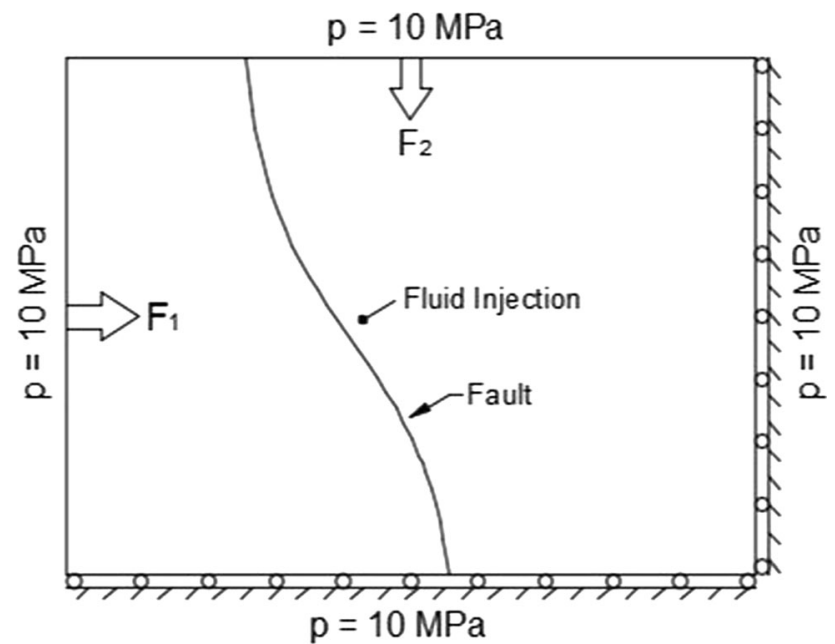

Fig. 16 Domain and boundary conditions for test case of the slip tendency of the fault under fluid injection

Table 2 Relevant parameters of case under fluid injection

\begin{tabular}{lll}
\hline Variable & Value & Description \\
\hline$d_{\Omega}$ & $1000 \mathrm{~m}$ & Depth \\
$\rho$ & $2670 \mathrm{~kg} / \mathrm{m}^{3}$ & Density \\
$\lambda, \mu$ & $32.04 \mathrm{GPa}$ & Lamé's parameters \\
$\mu_{0}$ & 0.6 & Reference friction coefficient \\
$V_{0}$ & $1.0 \mathrm{e}-6 \mathrm{~m} / \mathrm{s}$ & Reference slip rate \\
$d_{c}$ & $0.008 \mathrm{~m}$ & Characteristic slip distance \\
$A$ & 0.015 & Empirical dimensionless constants \\
$B$ & 0.019 & Empirical dimensionless constants \\
$V_{\text {linear }}$ & $1.0 \mathrm{e}-12$ & A cutoff for a linear slip rate dependence \\
$\phi$ & 0.1 & Porosity of the domain \\
$b$ & 1.0 & Biot coefficient \\
$M$ & $1.0 \mathrm{e} 8$ & Biot modulus \\
\hline
\end{tabular}

the left boundary we apply the traction. The relevant parameters are in Table 2. For the fluid flow problem, the initial pressure in the porous medium is $10^{7} \mathrm{~Pa}$, and the four boundaries are all Neumann boundaries with imposed pressure $10^{7} \mathrm{~Pa}$. The permeability tensor of the domain is

$$
\mathbf{K}=\left[\begin{array}{cc}
10^{-11} & 0 \\
0 & 10^{-11}
\end{array}\right] \mathrm{m}^{2} /(\text { Pa s }) \text {. }
$$

The thickness of the fault is $0.2 \mathrm{~m}$, and the permeability in the tangential direction and the normal direction is $10^{-13} \mathrm{~m}^{2} /(\mathrm{Pas})$. At the two fault tips, we impose pressure $p=10^{7} \mathrm{~Pa}$. We inject fluid on the node $(1720,1480)$ with a volume source term $0.00035 \mathrm{~m}^{3} / \mathrm{s}$. Each time step is 1 year. We consider two cases:

In these two cases, the stress on the left boundary and the front boundary all equal to $\mathbf{F}_{1}=\mathbf{F}_{2}=55 \mathrm{MPa}$. In the first case, the dip of the fault is $80^{\circ}$, while in the second case, the dip of the fault is $81^{\circ}$. The corresponding friction coefficient $\mu_{f}$ and slip tendency $\left|\tau^{\prime}(\mathbf{u}(\mathbf{x}))\right| /\left|\sigma_{\mathrm{n}}^{\prime}(\mathbf{u}(\mathbf{x}))\right|$ on the fault are shown in figures. In the first case, the results are shown in Fig. 17, the fault begins to slip after the 6th step. While in the second case, the results are shown in Fig. 18, the fault begins to slip after the 17th step. This difference is due to the different dip angles: vertical faults are less likely to slip with respect to inclined ones for the same imposed compressive stress. Besides, after the fault slips, in the slipping period, we set each time step last $0.02 \mathrm{~s}$, then in the first case, the fault totally slips 21 steps which last $0.42 \mathrm{~s}$, while in the second case, the fault totally slips 19 steps which lasts $0.38 \mathrm{~s}$. Here we could get the fault slipping distance, after we research on the numerical method in the three-dimensional case, we could get the fault slipping area, then we could calculate the magnitude of the earthquake induced by the fault reactivation, which will be a very interesting thing.

In order to analyze the effect of the permeability of the fault, we consider two more cases, setting the fault permeability to $10^{-14} \mathrm{~m}^{2} /(\mathrm{Pa} \mathrm{s})$. Other parameters are all the
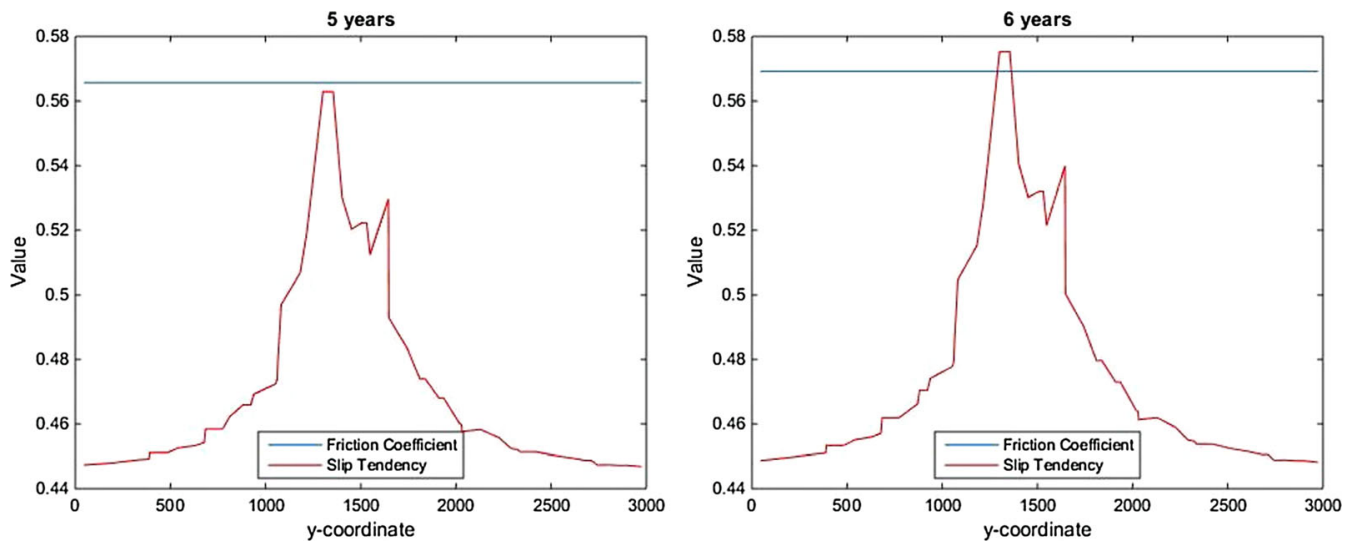

Fig. 17 Friction coefficient and slip tendency on the fault after 5 years and 6 years 

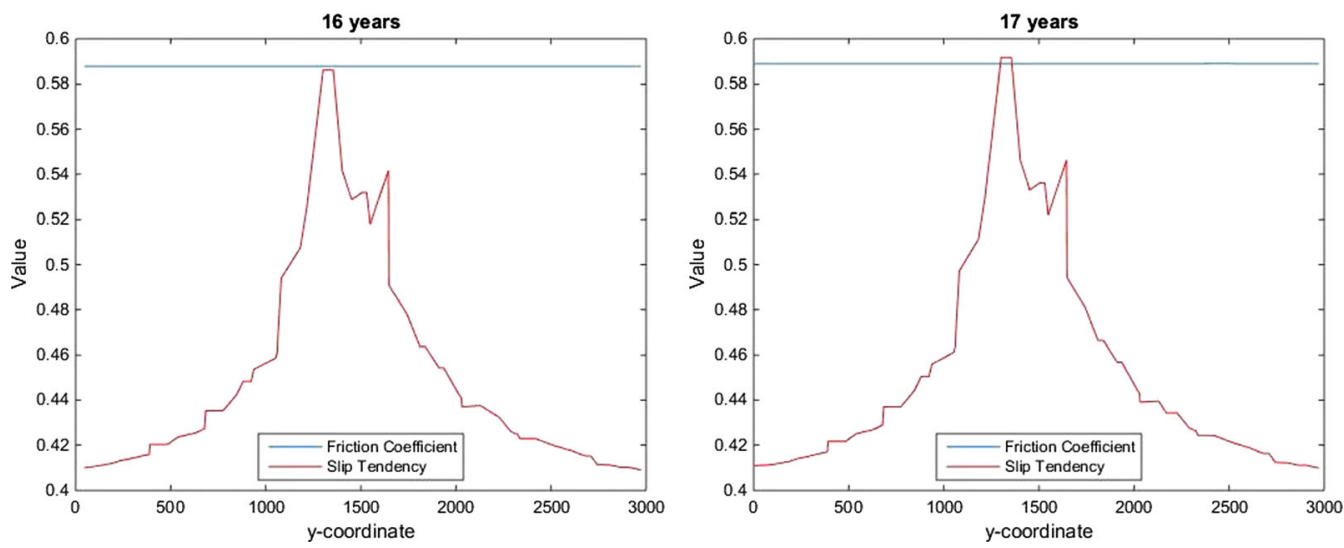

Fig. 18 Friction coefficient and slip tendency on the fault after 16 years and 17 years
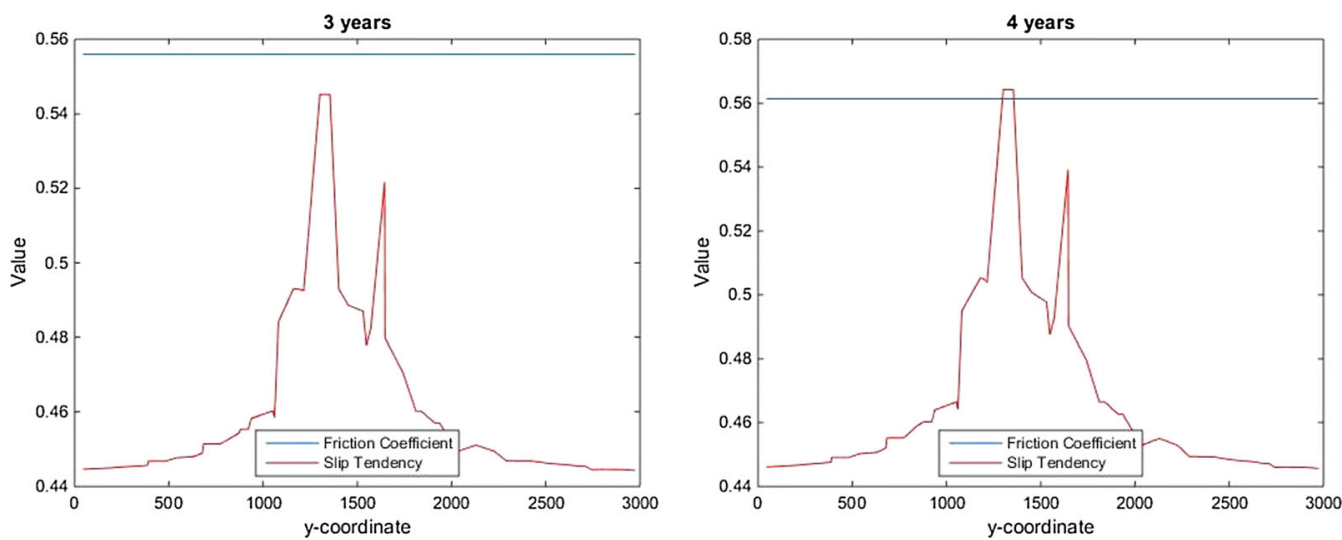

Fig. 19 Friction coefficient and slip tendency on the fault after 3 years and 4 years
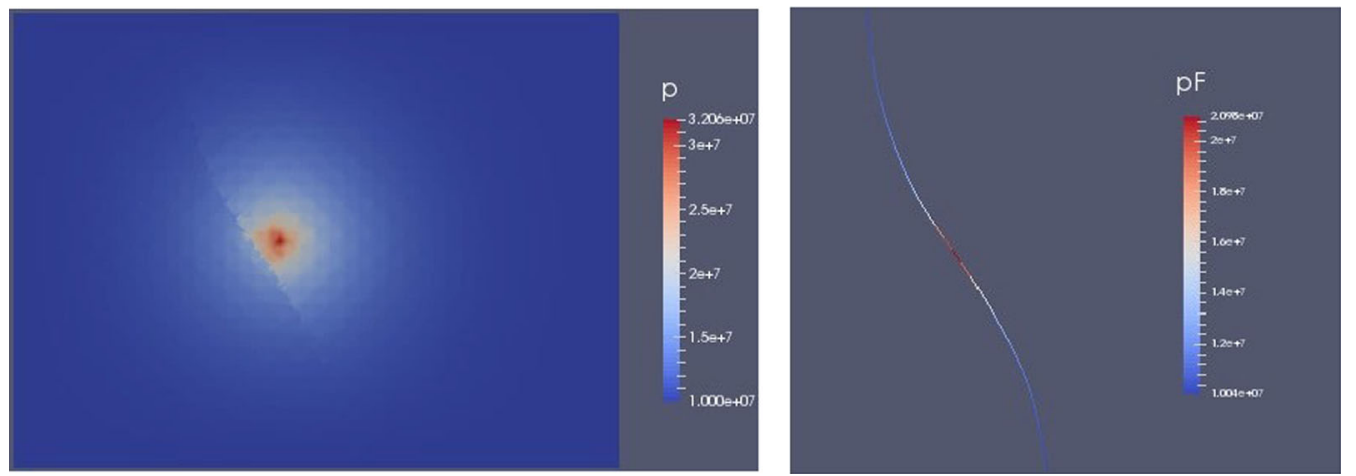

Fig. 20 The fluid pressure and the fluid pressure in the fault after 11 years

same as before. For a dip of the fault of $80^{\circ}$, the results are shown in Fig. 19, the fault begins to slip after the 4th step. While in the other case, when the dip of the fault is $81^{\circ}$, the results are shown in Fig. 21, the fault begins to slip after the 12th step, the fluid pressure and the fluid pressure in the fault after 11th step are shown in Fig. 20. In both cases, the fault fails earlier with respect to the previous case. A lower permeability of the fault leads to high pressures close to the injection point faster than a more permeable interface; pressure reduces the effective normal stress causing failure.

In order to analyze the effect of the position of the fluid injection, we repeat the previous cases keeping the same 

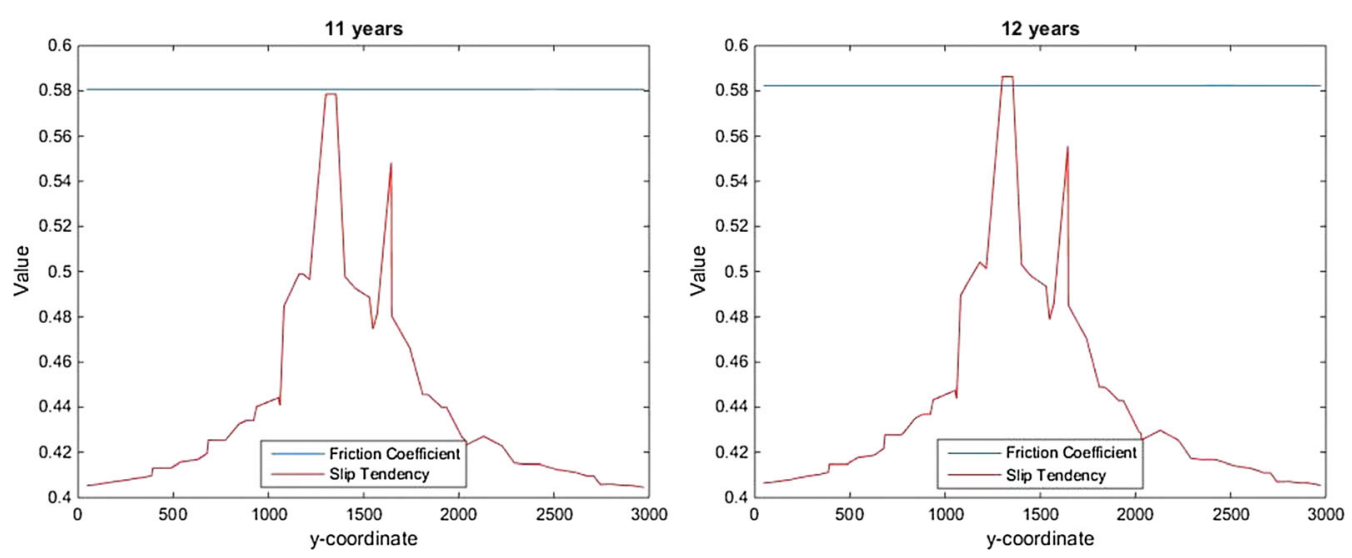

Fig. 21 Friction coefficient and slip tendency on the fault after 11 years and 12 years
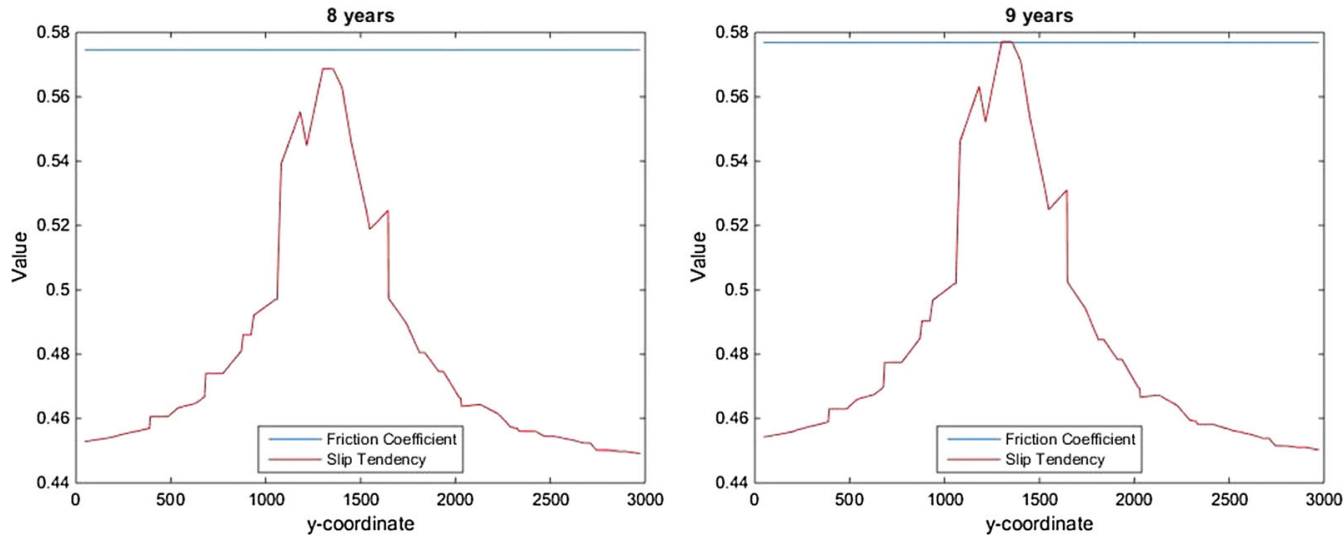

Fig. 22 Friction coefficient and slip tendency on the fault after 8 years and 9 years
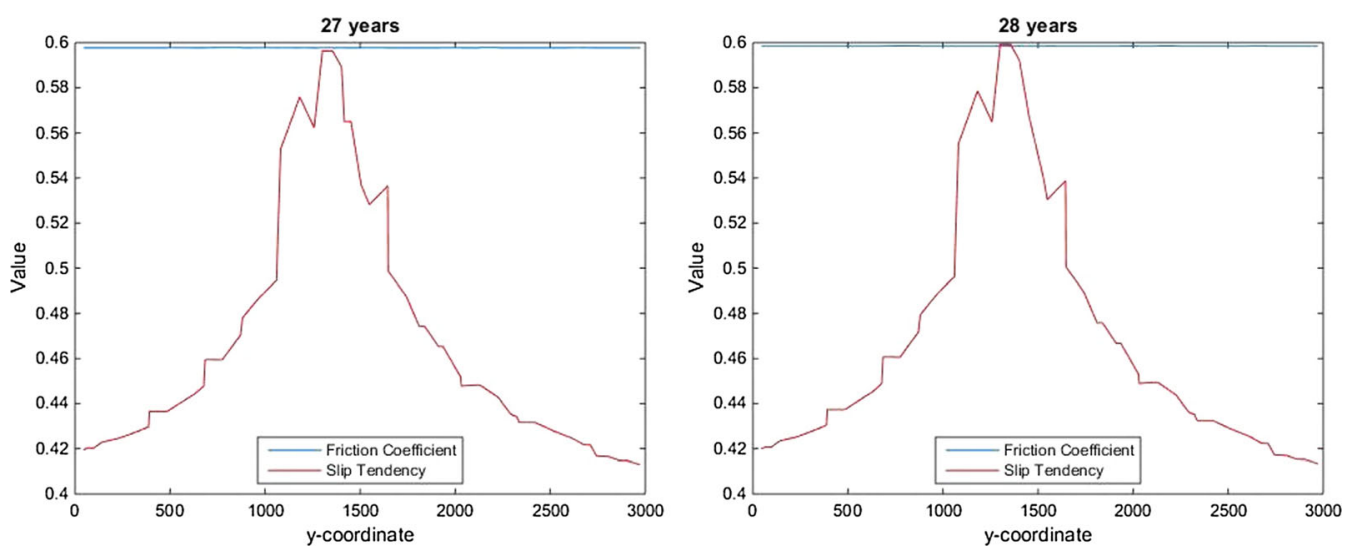

Fig. 23 Friction coefficient and slip tendency on the fault after 27 years and 28 years

physical parameters and changing only the injection point, which is now the point of coordinates $(1760,1450)$. The results for $80^{\circ}$ dip are shown in Fig. 22, the fault begins to slip after the 9 th step, while in the other case (dip of $81^{\circ}$ ), the results are shown in Fig. 23, the fault begins to slip after the 28th step. In this case, the distance of the injection point from the fault is larger, so the onset of slip is delayed.

\section{Conclusions}

In this work, we proposed a numerical method used to analyze the slip tendency of the fault under fluid injection based on the eXtended Finite Element Method used both for fluid and poroelasticity. We have imposed the dynamic coupling condition for the structural problem via a Nitsche 
penalization. Thanks to the eXtended Finite Element Method, we can accurately represent discontinuities in stress, displacements, fluid pressure and fluid velocity across straight and curved faults.

In order to study the slipping behavior of the fault in the process of reactivation, our research viewed the fault as a zero-thickness interface. Besides, we included a reduced model of the fluid flow in the fault which allows us to account for the hydraulic behavior in the fault and its effect on the surrounding medium.

We particularly focused on the map-view two-dimensional case, while when considering the depth and the dip of the fault, we regarded the system as a two-and-one-halfdimensional system based on Anderson's fault theory which assumes the vertical direction as principal stress component. We used the rate- and state-dependent friction model as the fault friction model, and Biot's theory of poroelasticity to study the coupling between fluid flow and mechanical deformation in porous media. Since the Fully Coupled Method between fluid flow and poromechanics is computationally expensive, we had investigated an Iteratively Coupled Method. In particular, we applied the socalled fixed-stress split. In such a scheme, the fluid flow problem was solved firstly by freezing the total means stress field, and then the results were used to solve the mechanical problem. In order to verify our method, some test cases were presented. For the coupling between fluid flow and geomechanics, we considered the Terzaghi Problem and the Mandel Problem, comparing our results with that of previously published works. While, for the mechanic problem, we compared the results with those obtained by using the software Pylith. From these test cases, we could know our method works well. Then we used our method to analyze one case about the slip tendency of the fault under fluid injection. From the results, we could know that the permeability of the fault could affect the slip tendency of the fault, the fault slips earlier in the case of smaller fault permeability than that in the case of larger fault permeability, besides, with the point injected fluid closer to the fault, the fault slips earlier. In the future, we will research on the method to simulate the earthquake and predict the corresponding earthquake magnitude after the fault slips. And then we will research on the permeability variation in the fault and the surrounding medium in the process of fault reactivation. Finally, we will focus on the three-dimensional case and study the corresponding numerical methods.

Acknowledgements LIU Daqing was supported by MOX in the Department of Mathematics of Politecnico di Milano and also supported by the China Scholarship Council (CSC) with a scholarship. This work was done mainly under Professor Luca Formaggia and Associate Professor Anna Scotti's guidance and their help. Luigi Vadacca also plays an important role in supporting this work. This work was implemented on the basis of the Getfem++ library, http:// download.gna.org/getfem $/ \mathrm{html} / \mathrm{homepage/.} \mathrm{Besides,} \mathrm{thanks} \mathrm{Franco}$ Dassi, Bianca Giovanardi, Alessio Fumagalli, Davide Baroli, Alberto Ferroni, Abramo Agosti, Stefano Zonca, Claudia Colciago, Mattia Penati, Francesca Mesolella, Marianna Signorini, and Daniele Rossi's help in the process of research.

Funding LIU Daqing has received funding by the China Scholarship Council (CSC) through a scholarship.

\section{Compliance with ethical standards}

Conflict of interest The author declares that he has no conflict of interest.

\section{References}

1. Aagaard BT, Heaton TH, Hall JF (2001) Dynamic earthquake ruptures in the presence of lithostatic normal stresses: implications for friction models and heat production. Bull Seismol Soc Am 91(6): 1765-1796

2. Aagaard B, Knepley M, Williams C, Strand L, Kientz S (2015) PyLith user manual version 2.1.0, 2015

3. Alboin C, Jaffré J, Roberts JE, Serres C (2001) Modeling fractures as interfaces for flow and transport in porous media. In: Fluid flow and transport in porous media: mathematical and numerical treatment (South Hadley, MA, 2001). Contemporary mathematics, American Mathematical Society, vol 295, no. 2002, pp 13-24

4. Anderson EM (1905) The dynamics of faulting. Trans Edinb Geol Soc 8:387-402

5. Anderson EM (1951) The dynamics of faulting and dyke formation with application to Britain. Oliver and Boyd, Edinburgh, p 1951

6. Annavarapu C, Hautefeuille M, Dolbow JE (2014) A Nitsche stabilized finite element method for frictional sliding on embedded interfaces. Part I: single interface. Comput Methods Appl Mech Eng 268:417-436

7. Annavarapu C, Settgast RR, Johnson SM, Fu P, Herbold EB (2015) A weighted Nitsche stabilized method for small-sliding contact on frictional surfaces. Comput Methods Appl Mech Eng 283:763-781

8. Armero F (1999) Formulation and finite element implementation of a multiplicative model of coupled poro-plasticity at finite strains under fully saturated conditions. Comput Methods Appl Mech Eng 171(3-4):205-241

9. Armero F, Simo JC (1992) A new unconditionally stable fractional step method for non-linear coupled thermomechanical problems. Int J Numer Methods Eng 35(4):737-766

10. Becker R, Burman E, Hansbo P (2009) A Nitsche extended finite element method for incompressible elasticity with discontinuous modulus of elasticity. Comput Methods Appl Mech Eng 198(41-44):3352-3360

11. Belytschko T, Black T (1999) Elastic crack growth in finite elements with minimal remeshing. Int $\mathrm{J}$ Numer Methods Eng 45(5):601-620

12. Berrone S, Pieraccini S, Scialò S (2013) On simulations of discrete fracture network flows with an optimization-based extended finite element method. SIAM J Sci Comput 35(2):908-935

13. Bevillon D, Masson R (2000) Stability and convergence analysis of partially coupled schemes for geomechanical-reservoir simulations. In: ECMOR VII-7th European conference on the mathematics of oil recovery, Baveno, Italy 
14. Biot MA (1941) General theory of three-dimensional consolidation. J Appl Phys 12(2):155-164

15. Both JW, Borregales M, Nordbotten JM, Kumar K, Radu FA (2017) Robust fixed stress splitting for Biot's equations in heterogeneousmedia. Appl Math Lett 68:101-108

16. Bouchon M, Streiff D (1997) Propagation of a shear crack on a nonplanar fault: a method of calculation. Bull Seismol Soc Am 87(1):61-66

17. Burman E, Zunino P (2011) Numerical approximation of large contrast problems with the unfitted Nitsche method. Lect Notes Comput Sci Eng 85:227-282

18. Burman E, Zunino P (2011) Numerical approximation of large contrast problems with the unfitted Nitsche method. In: Blowey J, Jensen M (eds) Frontiers in numerical analysis-Durham 2010. Lecture notes in computational science and engineering, vol 85. Springer, Berlin, Heidelberg

19. Cappa F, Rutqvist J (2011) Modeling of coupled deformation and permeability evolution during fault reactivation induced by deep underground injection of $\mathrm{CO}_{2}$. Int $\mathrm{J}$ Greenh Gas Control 5(2):336-346

20. Cappa F, Rutqvist J (2011) Modeling of coupled deformation and permeability evolution during fault reactivation induced by deep underground injection of $\mathrm{CO}_{2}$. Int $\mathrm{J}$ Greenh Gas Control 5(2):336-346

21. Chin LY, Raghavan R, Thomas LK (1998) Fully coupled geomechanics and fluid-flow analysis of wells with stress-dependent permeability. SPE 48857 5(1):32-45

22. Coon ET (2010) Nitsche extended finite element methods for earthquake simulation, Ph.D. thesis. Columbia University

23. Coon ET, Shaw BE, Spiegelman M (2011) A Nitsche-extended finite element method for earthquake rupture on complex fault systems. Comput Methods Appl Mech Eng 200(2011):2859-2870

24. Coussy O (1995) Mechanics of porous continua. Wiley, Chichester

25. D'Angelo C, Scotti A (2011) A mixed finite element method for Darcy flow in fractured porous media with non-matching grids. ESAIM Math Model Numer Anal ESAIM: M2AN 46(2):465-489. https://doi.org/10.1051/m2an/2011148

26. Dean RH, Gai X, Stone CM, Minkoff SE (2006) A comparison of techniques for coupling porous flow and geomechanics. SPE79709-PA 2006 Soc Pet Eng SPE 11(01):132-140

27. Del Pra M, Fumagalli A, Scotti A (2017) Well posedness of fully coupled fracture/bulk Darcy flow with XFEM. SIAM J Numer Anal 55(2):785

28. Dieterich JH (1981) Constitutive properties of faults with simulated gouge. In: Mechanical behavior of crustal rocks: the Handin volume, geophysical monograph series. AGU, Washington, vol 24, pp 108-120

29. Dieterich JH (1979) Modeling of rock friction: 1. Experimental results and constitutive equations. J Geophys Res Solid Earth 84(B5):2161-2168

30. Dolbow J, MoÃńs N, Belytschko T (2001) An extended finite element method for modeling crack growth with frictional contact. Comput Methods Appl Mech Eng 190(51-52):6825-6846

31. Duan B, Oglesby DD (2007) Nonuniform prestress from prior earthquakes and the effect on dynamics of branched fault systems. J Geophys Res Solid Earth 112(B5):1-18

32. Fries TP (2007) A corrected XFEM approximation without problems in blending elements. Int $\mathrm{J}$ Numer Methods Eng 75(5):503-532

33. Frih N, Martin V, Roberts JE, Saada A (2012) Modeling fractures as interfaces with nonmatching grids. Comput Geosci 16(4):1043-1060

34. Fumagalli A (2012) Numerical modelling of flows in fractured porous media by the XFEM method, Ph.D. thesis. Politecnico di Milano
35. Hansbo A, Hansbo P (2002) An unfitted finite element method, based on Nitsche's method, for elliptic interface problems. Comput Methods Appl Mech Eng 191(47-48):5537-5552

36. Hansbo A, Hansbo P (2002) An unfitted finite element method, based on Nitsche's method, for elliptic interface problems. Comput Methods Appl Mech Eng 191(47-48):5537-5552

37. https://geodynamics.org/cig/software/pylith/

38. Jha B, Juanes R (2014) Coupled multiphase flow and poromechanics: a computational model of pore pressure effects on fault slip and earthquake triggering. Water Resour Res 50:3776-3808. https://doi.org/10.1002/2013WR015175

39. Kame N, Yamashita $T$ (1999) Simulation of the spontaneous growth of a dynamic crack without constraints on the crack tip path. Geophys J Int 139(2):345-358

40. Kim J, Tchelepi H, Juanes R (2009) Stability, accuracy, and efficiency of sequential methods for coupled flow and geomechanics. SPE-119084-PA 2009 Soc Pet Eng SPE 16(02):249-262

41. Koller MG, Bonnet M, Madariaga R (1992) Modelling of dynamical crack propagation using time-domain boundary integral equations. Wave Motion 16(4):339-366

42. Lackner KS (2003) A guide to $\mathrm{CO}_{2}$ sequestration. Science 300(5626):1677-1678

43. Liu F, Borja RI (2008) A contact algorithm for frictional crack propagation with the extended finite element method. Int J Numer Methods Eng 76(10):1489-1512

44. Liu F, Borja RI (2009) An extended finite element framework for slow-rate frictional faulting with bulk plasticity and variable friction. Int J Numer Anal Methods Geomech 33(13):1535-1560

45. Marone C (1998) Laboratory-derived friction laws and their application to seismic faulting. Ann Rev Earth Planet Sci 26:643-696

46. Martin V, Jaffré J, Roberts JE (2005) Modeling fractures and barriers as interfaces for flow in porous media. Soc Ind Appl Math 26(5):1667-1691

47. Metz B, Davidson O, De Coninck H, Loos M, Meyer L (2005) IPCC special report on carbon dioxide capture and storage. Cambridge University Press, New York

48. Moës N, Dolbow J, Belytschko T (1999) A finite element method for crack growth without remeshing. Int $\mathrm{J}$ Numer Methods Eng 46(1):131-150

49. Nitsche J, Br FI (1970) Über ein Variationsprinzip zur Lösung von Dirichlet-Problemen bei Verwendung von Teilräumen, die keinen Randbedingungen unterworfen sind. Abhandlungen aus dem Mathematischen Seminar der Universitt Hamburg 36:9-15

50. Orr FM Jr (2009) Onshore geologic storage of $\mathrm{CO}_{2}$. Science 325:1656-1658

51. Pacala S, Socolow R (2004) Stability wedges: solving the climate problem for the next 50 years with current technologies. Science 305:968-972

52. Park KC (1983) Stabilization of partitioned solution procedure for pore fluid-soil interaction analysis. Int J Numer Methods Eng 19(11):1669-1673

53. Prévost J-H, Sukumar N (2016) Faults simulations for three-dimensional reservoir-geomechanical models with the extended finite element method. J Mech Phys Solids 86:1-18

54. Rodrigo C, Hu X, Ohm P, Adler JH, Gaspar FJ, Zikatanov LT (2017) New stabilized discretizations for poroelasticity and the Stokes' equations, pp 1-20. arXiv:1706.05169v2

55. Ruina A (1983) Slip instability and state variable friction laws. J Geophys Res 88(B12):10359-10370

56. Rutqvist J, Rinaldi AP, Cappa F, Moridis GJ (2013) Modeling of fault reactivation and induced seismicity during hydraulic fracturing of shale-gas reservoirs. J Pet Sci Eng 107:31-44

57. Samier P, Onaisi A, De Gennaro S (2008) A practical iterative scheme for coupling geomechanics with reservoir simulation. SPE-107077-PA 2008 Soc Pet Eng SPE 11(05):892-901 
58. Scholz CH (1989) Mechanics of faulting. Ann Rev Earth Planet Sci 17:309-334

59. Schwenck N, Flemisch B, Helmig R, Wohlmuth B (2015) Dimensionally reduced flow models in fractured porous media: crossings and boundaries. Comput Geosci 19(6):1219-1230

60. Settari A, Walters DA (2001) Advances in coupled geomechanical and reservoir modeling with applications to reservoir compaction. SPE-74142-PA 2001 Soc Pet Eng SPE 6(03):334-342

61. Shaw BE, Dieterich JH (2007) Probabilities for jumping fault segment stepovers. Geophys Res Lett 34(1):1-7

62. Shaw BE (2004) Self-organizing fault systems and self-organizing elastodynamic events on them: geometry and the distribution of sizes of events. Geophys Res Lett 31(17):1-4

63. Shaw BE, Rice JR (2000) Existence of continuum complexity in the elastodynamics of repeated fault ruptures. J Geophys Res 105(B10):23791-23810

64. Szulczewski ML, MacMinn CW, Herzog HJ, Juanes R (2012) Lifetime of carbon capture and storage as a climate-change mitigation technology. Proc Natl Acad Sci USA 109(14):5185-5189. https://doi.org/10.1073/pnas.1115347109
65. Tada T, Yamashita T (1997) Non-hypersingular boundary integral equations for two-dimensional non-planar crack analysis. Geophys J Int 130(2):269-282

66. Thomas LK, Chin LY, Pierson RG, Sylte JE (2003) Coupled geomechanics and reservoir simulation. SPE J 8(4):350-358

67. Ucar E, Berre I, Keilegavlen E (2015) Simulation of slip-induced permeability enhancement accounting for multiscale fractures. In: Proceedings: fortieth workshop on geothermal reservoir engineering

68. Wang HF (2000) Theory of linear poroelasticity. Princeton University Press, Princeton

69. Zienkiewicz OC, Paul DK, Chan AHC (1988) Unconditionally stable staggered solution procedure for soil-pore fluid interaction problems. Int J Numer Methods Eng 26(5):1039-1055

Publisher's Note Springer Nature remains neutral with regard to jurisdictional claims in published maps and institutional affiliations. 